\title{
Fungos macroscópicos do Pantanal do Rio Negro, Mato Grosso do Sul, Brasil
}

Vera Lucia Ramos Bononi ${ }^{1,2,3}$, Ademir Kleber Morbeck de Oliveira ${ }^{1}$, Josiane Ratier de Quevedo ${ }^{1}$ e Adriana de Mello Gugliotta ${ }^{2}$

Recebido: 12.07.2007; aceito: 01.10.2008

ABSTRACT - (Macroscopic fungi at the lowlands of Rio Negro, Mato Grosso do Sul, Brazil). The wetlands' biodiversity is virtually unknown, particularly to fungi. As a consequence of the devastation caused by the advances made by the cattle-raising industry, a significant part of the native vegetation has been eliminated and only fragments of savannah woods, vast grazing areas and furrows formed by the accumulation of logs cut have been left. At Rio Negro lowlands, five fungi collection excursions were carried out in 2006, in the driest period. Fifty six species of Basidiomycetes and one of macroscopic Ascomycetes were identified. All species are being mentioned for the first time for the State of Mato Grosso do Sul and Collybia bakeri Dennis, Entoloma cerussatum Pegler, Epithelopsis fulva (G. Cunn.) Jülich, Hypochniciellum subillaqueatum (Litsch.) Hjortstam, Hypochnicium vellereum (Ellis \& Cragin) Parmasto, Lentinus concavus (Berk.) Corner, Mycena parabolica (Fr.) Quél., Mycoaciella bispora (Stalpers) J. Erikss. \& Ryvarden, Nigroporus macroporus Ryvarden \& Iturr., Nothopanus hygrophanus (Mont.) Singer, Pholiota polychroa (Berk.) A.H. Sm. \& H.J. Brodie, Pleurotus agaves Dennis, Trametes subectypus (Murrill) Gilbn. \& Ryvarden, and Tricholomopsis tropica Dennis for the first time to Brazil. Key words: Ascomycetes, Basidiomycetes, biodiversity, Paraguay Basin

RESUMO - (Fungos macroscópicos do Pantanal do Rio Negro, Mato Grosso do Sul, Brasil). A biodiversidade do Pantanal é praticamente desconhecida, principalmente em relação aos fungos. Em consequiência da devastação pelo avanço da pecuária, grande parte da vegetação natural em áreas de fácil acesso foi suprimida, restando fragmentos de cerrado, extensas pastagens e leiras formadas pelo acúmulo da madeira derrubada. Em alguns locais, como na região do Pantanal do Rio Negro, parte da vegetação nativa foi relativamente preservada e nela, durante o ano de 2006, foram realizadas cinco excursões de coleta de fungos, nos períodos mais secos. Cinqüenta e seis espécies de Basidiomycetes e uma de Ascomycetes macroscópicos foram identificadas. Todas as espécies estão sendo citadas pela primeira vez para o Estado de Mato Grosso do Sul e região do Pantanal, e Collybia bakeri Dennis, Entoloma cerussatum Pegler, Epithelopsis fulva (G. Cunn.) Jülich, Hypochniciellum subillaqueatum (Litsch.) Hjortstam, Hypochnicium vellereum (Ellis \& Cragin) Parmasto, Lentinus concavus (Berk.) Corner, Mycena parabolica (Fr.) Quél., Mycoaciella bispora (Stalpers) J. Erikss. \& Ryvarden, Nigroporus macroporus Ryvarden \& Iturr., Nothopanus hygrophanus (Mont.) Singer, Pholiota polychroa (Berk.) A.H. Sm. \& H.J. Brodie, Pleurotus agaves Dennis, Trametes subectypus (Murrill) Gilbn. \& Ryvarden e Tricholomopsis tropica Dennis pela primeira vez para o Brasil.

Palavras-chave: Ascomycetes, Bacia do Alto Paraguai, Basidiomycetes, biodiversidade

\section{Introdução}

O Brasil é conhecido como detentor de uma das mais altas biodiversidades do mundo. Entretanto, informações existentes sobre a diversidade biológica são restritas a alguns tipos de organismos, como plantas superiores e vertebrados, e se encontram dispersas em instituições, museus e coleções científicas do país e do exterior.

O primeiro documento a centrar informações sobre a biodiversidade global, incluindo a brasileira, foi elaborado por ocasião da Conferência Rio92 (Groombridge 1992), seguido do trabalho de Mittermeier et al. (1998) que relacionaram espécies de plantas superiores, vertebrados e alguns invertebrados.

Os balanços sobre a biodiversidade brasileira feitos no Brasil (Bicudo \& Menezes 1996, Canhos 1997, Joly \& Bicudo 1998) avaliaram em cerca de 2.500 o número de espécies de fungos macroscópicos, nenhuma delas registrada para a região do Pantanal ou para o Estado do Mato Grosso do Sul.

\footnotetext{
1. Universidade para o Desenvolvimento do Estado e da Região do Pantanal-UNIDERP, Caixa Postal 2153, 79003-010 Campo Grande, MS, Brasil

2. Instituto de Botânica, Caixa Postal 3005, 01061-970 São Paulo, SP, Brasil

3. Autor para correspondência: vbononi@uol.com.br
} 
O Pantanal, localizado na Bacia do Alto Paraguai, é uma região de importância estratégica na administração dos recursos hídricos do Brasil, Bolívia e Paraguai. Representa uma das maiores extensões de áreas alagadas do planeta, sendo elo de ligação entre o Cerrado brasileiro e o Chaco da Bolívia e do Paraguai, entre os paralelos $16-22^{\circ} \mathrm{S}$ e os meridianos $55-58^{\circ} \mathrm{W}$. Considerada uma das maiores planícies de inundação da América Latina, ocupa cerca de 150.000 $\mathrm{km}^{2}$, e é formada pela junção dos rios da Bacia (rio Paraguai e seus afluentes), constituindo uma série de sub-bacias, constituídas por outros rios e córregos numa complexa rede hidrográfica. Esta região é periodicamente alagada durante os meses de outubro a março devido às chuvas intensas que normalmente ocorrem nos planaltos que cercam a região pantaneira, gerando um caráter plurianual de ciclos de enchentes e "secas" que na realidade são períodos de pequeno alagamento (Adamoli 1982).

A posição quase central da região pantaneira na América do Sul permite o encontro de diferentes províncias fitogeográficas, que são: ao norte, a Amazônia; a leste, Cerrado; ao sul, as Florestas Meridionais e a oeste, o Chaco Boliviano e Paraguaio, propiciando grande variedade de fitofisionomias, uma das razões que levou à criação do termo "pantanais" (Adamoli 1982).

De acordo com Pott \& Pott (2000), o Pantanal e a Bacia do Alto Paraguai são dominados, por espécies vegetais com ampla dispersão geográfica, encontradas em diferentes ecossistemas. O Bioma Cerrado ocupa cerca de $70 \%$ da região, seguido pelo Bioma da Floresta Amazônica, encontrado principalmente no Pantanal do Paraguai, representando $21 \%$ da vegetação da área (Adamoli 1982, Silva et al. 2000).

Devido à grande biodiversidade de espécies e ambientes, o Pantanal foi declarado Patrimônio Nacional pela Constituição Brasileira em 2000, reconhecido como Reserva da Biosfera Mundial e considerado um dos "hotspots" de biodiversidade e de recursos hídricos do planeta em razão de sua importância e do grau de ameaças ambientais que vem sofrendo (Mittermeier et al. 1998).

No Brasil o conhecimento dos fungos se concentra em regiões onde existem Universidades e Institutos de Pesquisa com micologistas, como a Amazônia, Pernambuco, São Paulo, Santa Catarina, Paraná e Rio Grande do Sul. Este é o primeiro levantamento de macromicetos de Mato Grosso do Sul (exceto pela monografia de conclusão do curso de Biologia de Josiane Ratier de Quevedo da Universidade para o Desenvolvimento do Estado e da Região do Pantanal-UNIDERP, co-autora deste trabalho). Para o Mato Grosso, seis espécies haviam sido citadas por Fidalgo (1968a). O objetivo deste trabalho é ampliar o conhecimento de fungos do Brasil, especificamente da região do Pantanal.

\section{Material e métodos}

Área de coleta - situada na planície de inundação do Rio Negro, na fazenda Santa Emília, localizada no quadrante geográfico $19^{\circ} 29^{\prime} 12,2^{\prime \prime}$ a $19^{\circ} 30^{\prime} 49,8^{\prime}$ 'S e $55^{\circ} 35^{\prime} 28,5^{\prime \prime}$ a $55^{\circ} 42^{\prime} 37,9^{\prime \prime} \mathrm{W}$ (figura 1), com 2.618 ha, onde se localiza o Instituto de Pesquisa do Pantanal (IPPAN) da UNIDERP e a Pousada Araraúna, a cerca de $250 \mathrm{~km}$ de distância de Campo Grande, com percursos via cidade de Rio Negro ou Aquidauana. O local apresenta uma variedade de ambientes que, de acordo com Adamoli (1982) e Pott \& Pott (2000), podem ser classificados como:

- "baía" - lagoa de água permanente ou temporária, de tamanho variado, com a presença de espécies aquáticas como camalote (Eichhornia spp.), chapéu-de-couro (Echinodorus spp.), florzeiro (Ludwigia spp.) e orelha-de-onça (Salvinia spp.);

- "capão" ou “caapão" - porção de mata, geralmente em elevações de 0,3 - $3 \mathrm{~m}$ acima da altura do campo, com formato elíptico ou circular e diâmetro variável de $5-100 \mathrm{~m}$, formando ilhas arbóreas, normalmente não inundáveis, podendo apresentar diferentes tipos de vegetação, como cerrado, cerradão ou mata e espécies vegetais diversas, tais como acuri (Scheelea phalerata Mart.), carandá (Copernicia alba Morong), jatobá (Hymenaea stigonocarpa Mart.), lixeira (Curatella americana L.), paratudo (Tabebuia aurea ( Manso) Benth. \& Hook.), pau-terra (Qualea parviflora Mart.) e piúva (Tabebuia ochracea (Cham.) Standl.);

- "cordilheira"- pequena elevação na planície (cerca de 1 a $3 \mathrm{~m}$ de altura), podendo ser resultado de antigos diques fluviais ou cordões arenosos ou argilosos, composta de vegetação de cerrado, cerradão ou mata, geralmente não inundável. Algumas espécies vegetais arbóreas encontradas são: aroeira (Myracrodruon urundeuva Allem.), angico-branco (Albizia niopioides (Spruce) Burkart), angicovermelho (Anaderanthera colubrina (Vell.) Brenan), cumbaru (Dipteryx alata Vogel), faveiro (Callistene fasciculata Mart.), cambará (Vochysia divergens Pohl), gonçalo-alves (Astronium fraxinifolium Schott), lixeira (Curatella americana L.) e tarumã (Vitex cymosa Bert.); 
- "corixo" - curso d'água estacional, com leito definido e mata ciliar descontínua, com a presença de diversas espécies de plantas aquáticas, como baceiro (Oxycaryum cubense (Poepp. \& Kunth) Palla), camalote (Eichhornia azurea (Swartz) Kunth), ervade-bicho (Polygonum spp.), lagartixa (Nymphaea amazonum Mart. \& Zucc.) e lodo (Utricularia spp.);

- "corixão" - pequeno rio intermitente, com canal definido e mata ciliar desenvolvida, que mantém água em trechos quando para de correr, podendo também ser o leito abandonado (braço) do próprio rio que lhe deu origem, apresentando diversas espécies de plantas aquáticas;

- "savana gramíneo-lenhosa" - que recebe outras denominações como campo, campo limpo, campo sujo ou campo alagado, sendo constituída normalmente de gramíneas e ervas. Pode ser campo inundado ou seco, cujas proporções alternam-se em função da precipitação local e/ou aporte de água pelos rios, sendo esta forma de vegetação mais associada ao fator drenagem do que à fertilidade do solo. Entre as espécies de gramíneas são encontradas capim-barbade-bode ou fura-bucho (Paspalum carinatum Humb. \& Bonpl. ex Feiige, P. lineare Trinius e Aristida spp.), capim-arroz (Oriza spp.), capim-de-cerrado (Andropogon spp.), grama-de-cerrado (Mesosetum spp.), mimosinho (Reimarochloa spp.), mimosopeludo (Paratheria prostrata Griseb.), além de espécies de Paspalum, Digitaria e Panicum.

A região já sofreu grandes desmatamentos e hoje é constituída por fragmentos de vegetação de cerrado e pastagens, sendo freqüente a presença de leiras de restos de árvores, formando pilhas de madeira que delimitam pastos. Nos restos vegetais, nas leiras e nos

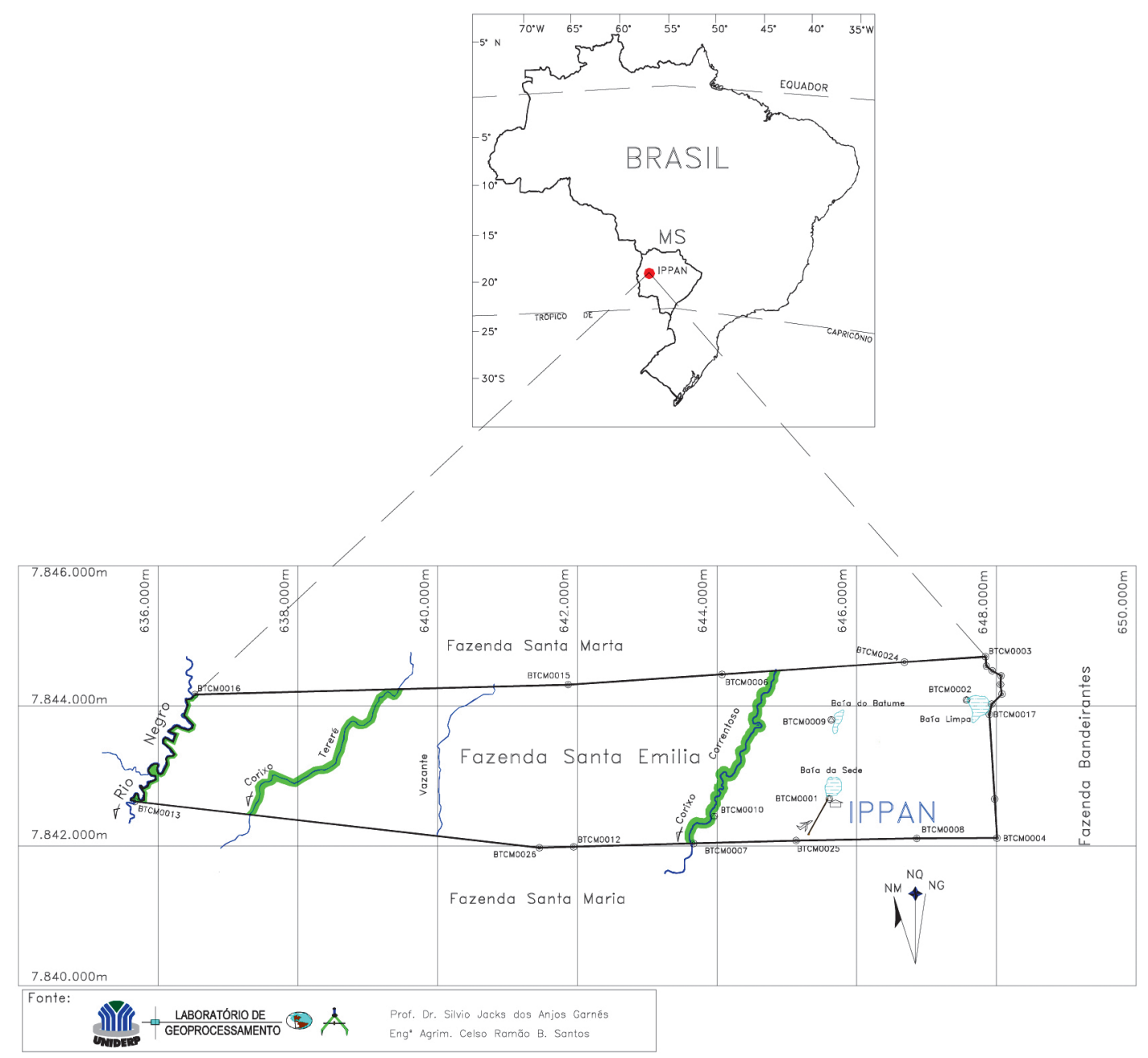

Figura 1. Estado do Mato Grosso do Sul e o Instituto de Pesquisas do Pantanal da Universidade para o Desenvolvimento do Estado e da Região do Pantanal, no Município de Rio Negro. 
gramados, é freqüente a observação de cogumelos em áreas não alagadas.

Clima e solos - o clima é considerado tropical, com classificação Aw (clima de savana segundo Köppen), com médias entre $23-25{ }^{\circ} \mathrm{C}$ e máximas e mínimas absolutas extremamente altas: de setembro a novembro, as máximas ultrapassam $40^{\circ} \mathrm{C}$; em maio, junho e julho, quando ocorrem entradas de frentes frias, as médias mínimas ficam abaixo de $20^{\circ} \mathrm{C}$. O inverno é seco (de maio até setembro, com os meses mais secos em julho e agosto) e verão chuvoso, sendo $80 \%$ das chuvas concentradas entre novembro e março, variando entre 1.000 a $1.400 \mathrm{~mm}$ por ano. A maior parte da região é coberta por solos hidromórficos $(92 \%)$, refletindo a drenagem insuficiente e a tendência para inundações periódicas, com composição arenosa (66\%).

Coleta e identificação - as excursões de coleta foram mensais, durante o ano de 2006, e tiveram a duração de 2 a 3 dias, procurando cobrir toda a área do IPPAN. Sempre que possível, foi utilizado GPS para georeferenciar os pontos de coleta. Os fungos foram coletados e secos conforme recomendado em Fidalgo
\& Bononi (1984) e a identificação foi feita no Instituto de Botânica de São Paulo, SP, com ajuda de chaves de identificação, observação de microestruturas e comparação com material de herbário. Todo material foi depositado no Herbário Científico "Maria Eneyda P. Kauffmann Fidalgo" (SP), do Instituto de Botânica. A classificação seguida segundo Kirk et al. (2001) pode ser consultada nas bases de dados CABI (http:// www.indexfungorum.org) e CBS (http://www.cbs. knaw.nl) onde também se encontram descrições das espécies. Apenas as espécies citadas pela primeira vez para o Brasil são descritas neste trabalho.

\section{Resultados e Discussão}

Foram identificadas 56 espécies de Basidiomycetes (Basidiomycota) e uma de Ascomycetes (Ascomycota). Todas as espécies estão sendo citadas pela primeira vez para o Estado de Mato Grosso do Sul e para a região do Pantanal.

As espécies estão listadas dentro dos respectivos grupos taxonômicos e por ordem alfabética.

Chave para a identificação de espécies de fungos macroscópicos do Pantanal

1. Fungo esférico ou semi-esférico, preto ou marrom muito escuro, com ascos e ascósporos

Daldinia concentrica

1. Fungo de outras formas que não esférica ou semi-esférica, de cores variadas, mas não totalmente preto, com basídios e basidiósporos

2. Basidioma clavarióide, muito ramificado, branco Lachnocladium brasiliense

2. Basidioma não clavarióide, não ramificado, de qualquer coloração

3. Superfície himenial com falsas lamelas que se enrolam aos pares formando cânulas radiais, coloração acinzentada Schizophyllum commune

3. Superfície himenial sem falsas lamelas, lisa, hidnóide, poróide ou lamelar

4. Superfície himenial lisa a hidnóide

5. Basidioma ressupinado

6. Basidioma amarelado a ocráceo; superfície himenial hidnóide

6. Basidioma branco a creme; superfície himenial não hidnóide

7. Medas grandes, conspícuas Mycoaciella bispora

7. Medas ausentes Epithelopsis fulva

8. Cistídios presentes, numerosos, cilíndricos a fusiformes

8. Cistídios ausentes Hypochnicium sphaerosporum

9. Basidioma bem aderido ao substrato; basídios freqüentemente irregulares com apenas um esporo; basidiósporo liso, com pequeno apículo, fracamente amilóide, 3,5-4,5 × 2,5 um

.Hypochniciellum subillaqueatum

9. Basidioma frouxamente preso ao substrato, basídios com mais de um esporo, basidiósporo irregularmente granulado, globoso, não amilóide, 7-8 $\mu \mathrm{m}$ diâm. 
5. Basidioma pileado a efuso-reflexo

10. Basidioma em forma de roseta, com diversos píleos fundidos; superfície abhimenial concentricamente zonada em diferentes tons de castanho e cinza Thelephora griseozonata

10. Basidioma séssil a efuso-reflexo; superfície abhimenial tomentosa, concentricamente zonada, ocrácea Stereum ostrea

4. Superfície himenial poróide ou lamelar

11. Basidioma estipitado, com píleo de margens enroladas para baixo, não permitindo a visualização de lamelas; superfície do píleo pilosa Montagnea haussknechtii

11. Basidioma estipitado ou não, com lamelas ou poros visíveis na superfície inferior do píleo; superfície do píleo pilosa ou não

12. Superfície himenial lamelar

13. Basidioma coriáceo, resistente

14. Basidioma séssil

Gloeophyllum striatum

14. Basidioma centralmente estipitado

15. Basidioma branco quando fresco; superfície abhimenial glabra

Lentinus concavus

15. Basidioma castanho; superfície abhimenial com escamas ou pelos

16. Superfície abhimenial pilosa, geralmente radialmente estriada Lentinus crinitus

16. Superfície abhimenial esquamulosa, não radialmente estriada, podendo tornar-se rugosa com a maturidade Lentinus swartzii

13. Basidioma carnoso, frágil

17. Estipe ausente ou excêntrico

18. Estipe excêntrico, robusto, com manchas marrom-avermelhadas na base Nothopanus hygrophanus

18. Estipe ausente ou excêntrico curto, sem manchas coloridas na base Pleurotus agaves

17. Estipe central bem desenvolvido

19. Esporada rósea; basidiósporos angulares Entoloma cerussatum

19. Esporada de cor variável, ocre, ferrugínea, castanha, creme ou branca, nunca rósea; basidiósporos não angulares (mas podendo ser rombóides)

20. Esporada ocre ou ferrugínea

21. Píleo amarelo-ovo; basidiósporo truncado, com poro de germinação conspícuo Bolbitius vitellinus

21. Píleo de coloração diferente, amarelo-claro, branco, creme, bege, sépia, castanho claro a escuro, castanhoalaranjado, castanho-rosado, castanho-acinzentado, castanho-ferrugíneo, a quase preto; basidiósporos nunca truncados, com ou sem poro de germinação

22. Basidiósporos com poro de germinação

23. Basidioma lignícola; píleo castanho-alaranjado

Pholiota polychroa

23. Basidioma terrestre; píleo de coloração creme com centro mais escuro, castanho Hypholoma trinitense

22. Basidiósporos sem poro de germinação

24. Basidiósporos com paredes ornamentadas, verrucosas Gymnopilus earlei

24. Basidiósporos com parede lisa Phaeomarasmius limulatellus 
20. Esporada de outra coloração, castanha, creme a branca, nunca ocre ou ferrugínea

25. Lamelas escuras adnatas ou decurrentes; basidiósporos castanho-escuros

26. Superfície pilear epitelial; píleo sépia; basidiósporos castanho-fuscos Psathyrella murrillii

26. Superfície pilear indiferenciada; píleo de coloração diferente; branco, creme, amarelo-claro, bege, castanhoamarelado a castanho-chocolate; basidiósporos castanho-esverdeados escuros

27. Píleo até $8 \mathrm{~cm}$ diâm.; estipe até $14 \mathrm{~cm}$ compr.; queilocistídios ramificados Psilocybe zapotecorum

27. Píleo geralmente menor que $1 \mathrm{~cm}$ diâm.; estipe até 1,5 cm compr.; queilocistídios não ramificados Psilocybe venezuelana

25. Lamelas adnatas ou livres; basidiósporos hialinos

28. Basidioma branco a bege com escamas escuras; superfície abhimenial do píleo geralmente viscosa; basidiósporos grandes, globosos a subglobosos, em torno de $22 \mu \mathrm{m}$ diâm. Oudemansiella canarii

28. Basidioma de qualquer coloração; superfície abhimenial do píleo não viscosa; basidiósporos elípticos ou globosos, sempre menores que $22 \mu \mathrm{m}$

29. Píleo amarelo-claro com escamas ou pêlos

30. Píleo campanulado, com escamas amareloacastanhadas; anel bem desenvolvido Leucocoprinus birnbaumii

30. Píleo aplanado, centralmente depresso, com pêlos na superfície; anel ausente .... Tricholomopsis tropica

29. Píleo liso sem escamas ou pêlos, castanho-rosado, castanho-acinzentado a castanho-escuro

31. Basidiomas agrupados; píleo campanulado, até 2,5 cm diâm.; estipe castanho-claro, mais escuro na base, até $3 \mathrm{~mm}$ diâm.

32. Basidiósporos $8 \times 4 \mu \mathrm{m}$ Mycena inclinata

32. Basidiósporos 8-10 $\times 6-7 \mu \mathrm{m}$ Mycena parabolica

31. Basidiomas agrupados ou solitários, píleo maior que 2,5 cm diâm.; estipe castanho-claro, maior que $3 \mathrm{~mm}$ diam.

33. Estipe maior que $5 \mathrm{~mm}$ diâm.; basidiósporos $6-8 \times 3-3,5 \mu \mathrm{m}$ Collybia neotropica

33. Estipe até $4 \mathrm{~mm}$ diâm.; basidiósporos $3-4,5 \times 2-2,5 \mu \mathrm{m}$ Collybia bakeri

12. Superfície himenial poróide

34. Basidioma estipitado

35. Basidioma lateralmente estipitado; superfície abhimenial lacada; basidiósporos globosos com parede dupla e ornamentada

Amauroderma renidens

35. Basidioma lateralmente a centralmente estipitado; superfície branca a creme, não laçada; basidiósporos com parede simples e lisa 
34. Basidioma ressupinado a séssil

36. Basidioma ressupinado

37. Superfície himenial branca a creme; poros regulares, tornando-se quase hidnóides em substratos inclinados; hifa generativa com ansa, reação xantocróica negativa Trechispora regularis

37. Superfície himenial castanho-ferrugínea; poros regulares; hifa generativa com septos simples; reação xantocróica positiva

Phellinus melleoporus

36. Basidioma efuso-reflexo a séssil, às vezes pseudoestipitado

38. Basidiósporos castanhos a amarelados, de parede dupla, com a parede externa lisa e a parede interna ornamentada; basidioma sempre lenhoso (muito duro)

39. Superfície abhimenial lacada

40. Superfície himenial inicialmente branca, castanha na maturidade, 3-4 poros $/ \mathrm{mm}$; basidiósporos 12-15 $\times 8-10 \mu \mathrm{m}$

Ganoderma oerstedtii

40. Superfície himenial creme, castanha quando tocada, 4-5 poros/mm; basidiósporos 9-12 × 5,5-8 $\mu \mathrm{m}$ Ganoderma lucidum

39. Superfície abhimenial opaca, não lacada

41. Superfície himenial amarelada, escurecendo (quase preta) quando tocada, 4-5 poros/mm; basidiósporos 9-12 × 7-9 $\mu \mathrm{m}$; crusta sem elementos clavados Ganoderma brownii

41. Superfície himenial creme a castanho-amarelada, escurecendo (marrom) quando tocada, 5-7 poros $/ \mathrm{mm}$; basidiósporos $10-12 \times 7-8 \mu \mathrm{m}$; crusta com elementos clavados Ganoderma lobatum

38. Basidiósporos hialinos a acastanhados, de parede simples, basidioma macio, flexível a coriáceo

42. Hifa generativa com septo simples

43. Basidioma fino, até $4 \mathrm{~mm}$ esp., não xantocróico; superfície abhimenial creme; superfície himenial creme-rosada a castanho-rosada; basidiósporos alantóides ..... Gloeoporus telephoroides

43. Basidioma até $5 \mathrm{~cm}$ esp., xantocróico; ambas as superfícies castanho-ferrugíneas; basidiósporos ovóides a elipsóides

44. Basidioma fibroso a coriáceo, de consistência macia; sistema hifálico monomítico Inonotus patouillardii

44. Basidioma coriáceo; sistema hifálico dimítico

45. Setas presentes, ventricosas Phellinus gilvus

45. Setas ausentes Phylloporia ribis

42. Hifa generativa com ansa

46. Poros grandes, $1-3 / \mathrm{mm}$

47. Cistídios incrustrados presentes

48. Basidioma com consistência de cortiça, macio, branco quando fresco, ocráceo quando seco; superfície abhimenial lanosa Echinoporia aculeifera

48. Basidioma coriáceo, flexível, castanho-escuro; superfície abhimenial com pêlos escuros e ramificados Trichaptum perrottetii

47. Cistídios incrustrados ausentes

49. Superfície abhimenial glabra; sistema hifálico dimítico Nigroporus macroporus

49. Superfície abhimenial vilosa, podendo tornar-se glabra na maturidade; sistema hifálico trimítico 
50. Superfície abhimenial vilosa, glabra na maturidade, concentricamente zonada, zonas purpúreas e castanhas, claras e escuras; basidiósporos cilíndricos, 9-14 × 4,5-5,5 $\mu \mathrm{m}$

Hexagonia variegata

50. Superfície abhimenial vilosa, concentricamente zonada, bege a castanho-clara; basidiósporos elipsóides, menores que $9 \mu \mathrm{m}$ compr.

51. Basidioma fino, até $2 \mathrm{~mm}$ esp.; tubos muito rasos com no máximo $1 \mathrm{~mm}$ compr.; superfície abhimenial bege a acinzentada na maturidade; superfície himenial cremeamarelada a bege; basidiósporos 4,8-7,2 $\times$ 2,4-3,6 $\mu \mathrm{m}$ Trametes villosa

51. Basidioma 2-7 mm esp.; tubos até $4 \mathrm{~mm}$ compr.; superfície abhimenial ocrácea a castanha; superfície himenial bege a castanha; basidiósporos 5-8,5 × 2,5-3,5 $\mu \mathrm{m}$ Coriolopsis polyzona

46. Poros pequenos, menos que $3 / \mathrm{mm}$

52. Basidioma macio e frágil, corticoso, branco a creme; sistema hifálico monomítico, hifas gelatinizadas em $\mathrm{KOH}$; basidiósporos alantóides Tyromyces fumideceps

52. Basidioma coriáceo a lenhoso, resistente, creme, castanho a alaranjado; sistema hifálico dimítico a trimítico, hifas não gelatinizadas em $\mathrm{KOH}$; basidiósporos não alantóides

53. Basidioma vermelho-alaranjado Pycnoporus sanguineus

53. Basidioma de coloração diferente, esbranquiçado, bege, acinzentado, ocráceo, castanho-escuro a enegrecido, nunca vermelho-alaranjado

54. Basidioma lenhoso; superfície abhimenial com crusta enegrecida; basidiósporos de parede espessa, piriformes a levemente truncados; cistídios presentes, ventricosos Perenniporia martii

54. Basidioma coriáceo; superfície abhimenial sem crusta enegrecida; basidiósporos de parede fina, elipsóides a cilíndricos; cistídios ausentes 55. Superfície abhimenial fimbriada, com pêlos ramificados, castanho-escuros aproximadamente $0,5 \mathrm{~cm}$ compr....Hexagonia hydnoides

55. Superfície abhimenial finamente tomentosa a glabra, nunca fimbriada

56. Basidioma até $2,5 \mathrm{~cm}$ esp. na base; superfície abhimenial bege a acinzentada, tornado-se ocrácea à partir da base; basidiósporos 6-7,2 ×2,4 $\mu \mathrm{m}$

Trametes cubensis

56. Basidioma fino, até $0,5 \mathrm{~cm}$ esp. na base; superfície abhimenial esbranquiçada; basidiósporos 3,5-5 × 1-2 $\mu \mathrm{m}$ 
Ascomycetes

\section{XYLARIACEAE}

Daldinia concentrica (Bolton) Ces. \& DeNot., Comm. Soc. crittog. Ital.: 1197. 1863.

Descrições e ilustrações: Viégas (1944), Rogers et al. (1999).

Material examinado: BRASIL. MATo GRosso do SUL: Rio Negro, Pantanal do Rio Negro, sobre madeira em decomposição, 25-VI-2006, V.L.R. Bononi et al. s.n. (SP381532).

Espécie cosmopolita (Perez-Silva 1973), com muitas fotos e descrições na internet, conhecida popularmente na América do Norte e Europa como "bolo-do-rei-artur". No Brasil a espécie foi referida para os Estados do Pará, Pernambuco, São Paulo e Rio Grande do Sul (Viégas 1944, Bezerra \& Maia 2006).

Basidiomycetes

\section{AGARICACEAE}

Leucocoprinus birnbaumii (Corda) Singer, Sydowia 15 (1-6): 67. 1962 = Agaricus birnbaumii Corda, Icon. Fung. 3: 48. 1839.

Descrições e ilustrações: Guzmán-Dávalos \& Guzmán (1982), Gimenes (2007).

Material examinado: BRASIL. Mato Grosso do Sul: Rio Negro, Pantanal do Rio Negro, 19³1'0,4'S e $55^{\circ} 38^{\prime} 0,5^{\prime}$ 'W, em solo, 24-VI-2006, V.L.R. Bononi et al. s.n. (SP381332).

Espécie cosmopolita, citada nas Américas para Venezuela, Bermudas, Martinica, Trinidad e Tobago (Pegler 1983a). No Brasil citada para o Estado de São Paulo, nos Parques Estaduais das Fontes do Ipiranga (Grandi et al. 1984, Gimenes 2007) e da Ilha do Cardoso (Capelari 1989) e no Rio Grande do Sul (Rick 1907, 1961, Pereira \& Putzke 1990). Espécie venenosa, comum em jardins e vasos.

Montagnea haussknechtii Rab., Fl. Scan.: 339. 1836. (2002).

Descrição e ilustrações: Baseia \& Milanez

Material examinado: BRASIL. Mato Grosso do Sul: Rio Negro, Pantanal do Rio Negro, corixo, 19³0'23”'S e 55³6'53,3'W, 25-VI-2006, T.C. Urigas s.n. (SP381338).
Espécie de ampla distribuição em vários continentes. No Brasil, citada para o Cerrado da Estação Ecológica de Jataí no Estado de São Paulo (Baseia \& Milanez 2002). Era classificada até recentemente entre os Gasteromycetes e pela biologia molecular foi considerado entre os basidiomicetos (Kirk et al. 2001).

\section{ATHELIACEAE}

Hypochniciellum subillaqueatum (Litsch.) Hjortstam, Mycotaxon 13: 126. $1981 \equiv$ Corticium subillaqueatum Litsch., Annls. mycol. 39: 128. 1941 = Leucogyrophana subillaqueata (Litsch.) Jülich., Persoonia 8: 56. 1974.

Basidioma ressupinado, liso, branco a creme; frouxamente ligado ao substrato, margem branca, esmaecente ou fibrilosa. Sistema hifálico monomítico; hifas generativas com ansas, pouco ramificadas, parede fina, 2-5 um diâm. Cistídios ausentes. Basídios clavados, freqüentemente vários saindo de uma hifa comum, 15-30 × 3-4,5 $\mu \mathrm{m}$, tetraesporados. Basidiósporos elipsóides, adaxialmente achatados, com pequeno apículo, hialinos, cianófílos, parede espessada e lisa, 3,5-4,5 × 2,5 um. Conídios não observados.

Descrição complementar e ilustrações: Eriksson \& Ryvarden (1976, como Leucogyrophana subillaqueata).

Material examinado: BRASIL. Mato Grosso do SuL: Rio Negro, Pantanal do Rio Negro, sobre tronco em decomposição, 26-VI-2006, V.L.R. Bononi et al. 29 (SP).

Espécie citada para Suécia e Finlândia (Eriksson \& Ryvarden 1976) e Estados Unidos (Gilbertson e Budington 1970). Constitui primeira citação para o Brasil.

\section{BOLBITIACEAE}

Bolbitius vitellinus (Pers. ex Fr.) Fr., Epicr. Syst. mycol.: 254. 1838 = Agaricus vitellinus Pers., Synopsis Methodica Fungorum: 402. 1801.

Descrição e ilustrações: Pegler (1983a).

Material examinado: BRASIL. Mato GRosso do Sul: Rio Negro, Pantanal do Rio Negro, 19³1'4,1'S e 55³7'59'”, sobre esterco, 24-VI-2006, V.L.R. Bononi et al. 21 (SP); idem, $19^{\circ} 30^{\prime} 0,7^{\prime \prime} \mathrm{S}$ e $55^{\circ} 36^{\prime} 32,1^{\prime \prime} \mathrm{W}$, sobre esterco, 24-VI-2006, V.L.R. Bononi et al. s.n. (SP381544). 
A espécie é cosmopolita (Pegler 1997) e geralmente ocorre sobre húmus, no solo, sendo freqüente em vários continentes. No Brasil, foi citada para os Estados de São Paulo (Pegler 1997) e Rio Grande do Sul (Pereira \& Putzke 1990). Na Venezuela, citada sobre esterco de asno (Dennis 1970).

\section{CORTINARIACEAE}

Gymnopilus earlei Murrill, Mycologia 5: 22. 1913.

Descrição e ilustrações: Capelari (1989).

Material examinado: BRASIL. Mato Grosso do Sul: Rio Negro, Pantanal do Rio Negro, $19^{\circ} 31^{\prime} 0,4^{\prime \prime} S$ e $55^{\circ} 38^{\prime} 0,5^{\prime}$ 'W, 24-III-2006, V.L.R. Bononi s.n. (SP381897).

Espécie descrita para Jamaica e encontrada no Brasil, na Ilha do Cardoso, no Estado de São Paulo (Capelari 1989). Meijer (2006) cita Gymnopilus cf. earlei para o Estado do Paraná.

Phaeomarasmius limulatellus Singer, Sydowia 7: 254. 1953. (1952).

Descrição e ilustrações: Singer \& Digilio

Material examinado: BRASIL. Mato Grosso do SuL: Rio Negro, Pantanal do Rio Negro, 19³0'33,9'S e 55³7'45”W, em gramado, 25-III-2006, A.K.M. de Oliveira \& J.R. Quevedo s.n. (SP381898).

Espécie com referência para Chile, Argentina e Brasil, no Rio Grande do Sul (Singer \& Digilio 1952, Pereira \& Putzke 1990).

\section{ENTOLOMATACEAE}

Entoloma cerussatum Pegler, Kew Bull., Addit ser. 9: 329. 1983.

Basidioma pileado; píleo plano-convexo com pequeno umbo, 5-8 cm diâm. Superfície abhimenial branca, higrófana, estriada; formada por hifas aglutinadas. Margem translúcida estriada. Lamelas adnato-sinuadas, brancas a castanho-claro-rosadas, com lamélulas de dois comprimentos; margem irregular. Estipe cilíndrico, sólido a fistuloso, branco, glabro, logitudinalmente estriado, 5-8 mm. Contexto $3 \mathrm{~mm}$ espesso. Trama regular; hifas hialinas, sem ansas, 3-17 um diâm. Cistídios ausentes. Basídios clavados, $40 \times 8 \mu \mathrm{m}$, tetraesporados. Basidiósporos heterodiamétricos, depresso em uma das faces, rosa, 8-10 um diâm.

Ilustrações: Pegler (1983a).

Material examinado: BRASIL. Mato Grosso do Sul: Rio Negro, Pantanal do Rio Negro, 19³0'11,4’'S e $55^{\circ} 36^{\prime} 32,8^{\prime} \mathrm{W}$, em gramado, isolado ou em pequenos grupos, 24-VI-2006, V.L.R. Bononi et al. s.n. (SP381535).

Espécie com citação para Martinica e Guadalupe, ocorrendo solitário ou em pequenos grupos (Pegler 1983a). Constitui primeira citação para o Brasil.

\section{EPITHELIACEAE}

Epithelopsis fulva (G. Cunn.) Jülich, Persoonia 8(4): 457. 1976 E Epithele fulva G. Cunn., Trans. of the Royal Soc of New Zealand 83: 631. 1956.

Basidioma ressupinado, membranoso bem aderido ao substrato, formando áreas irregulares, branco a ocre, 8-10 × 1-3 cm. Margem aracnóide, concolor. Contexto até 200 um esp.; monomítico, hifas generativas hialinas, com ansas, 3-6 um diâm. Medas presentes, até $100 \mu \mathrm{m}$ altura. Basídios cilíndricos, mais largos na base, 25-30 × 6-8 $\mu \mathrm{m}$, tetraesporados. Basidiósporos elipsóides a globosos, hialinos, de parede fina, lisa, 10-12 × 5-4 $\mu \mathrm{m}$.

Ilustrações: Cunningham (1963), Jülich (1976).

Material examinado: BRASIL. Mato Grosso do Sul: Rio Negro, Pantanal do Rio Negro, sobre madeira em decomposição, 19³0'57,4"'S e 55 37'49,4’'W, 24-III2006, V.L.R. Bononi s.n. (SP 381913).

Espécie descrita para Nova Zelândia por Cunningham (1963). Constitui primeira citação para o Brasil.

\section{GANODERMATACEAE}

Amauroderma renidens (Bres.) Torrend, Broteria, ser. bot. 18: 136. 1920 = Ganoderma renidens Bres., Hedwigia 35: 380. 1896

Descrições e ilustrações: Furtado (1981), Ryvarden (2004).

Material examinado: BRASIL. Mato Grosso Do Sul: Rio Negro, Pantanal do Rio Negro, $19,49^{\circ} \mathrm{S}$ e $55,62^{\circ} \mathrm{W}$, sobre tronco caído muito decomposto, 25 VI-2006, V.L.R. Bononi 31 (SP).

A espécie só era conhecida para o Estado de Santa Catarina, localidade-tipo (Furtado 1981, Ryvarden 2004) 
Ganoderma brownii (Murrill) Gilbn., Mycologia 53: 405. 1962 三Elfvingia brownii Murrill, Western Polypores 5: 29. 1915. (1986).

Descrição e ilustrações: Gilbertson \& Ryvarden Material examinado: BRASIL. Mato Grosso do Sul: Rio Negro, Pantanal do Rio Negro, sobre madeira em decomposição, 25-III-2006, A.K.M. de Oliveira \& J.R. Quevedo s.n. (SP381326).

Espécie citada para Califórnia, Estados Unidos da América (Gilbertson \& Ryvarden 1986) e sudeste da América do Sul, incluindo Brasil, sem especificação de localidade (Gottlieb et al. 2000).

Ganoderma lobatum (Schwein.) G.F. Atk., Annls mycol. 6: 190. 1908 = Polyporus lobatus Schwein., Trans. Am. Phill. Soc., New Series, 4: 157. 1832. (1986).

Descrição e ilustrações: Gilbertson \& Ryvarden

Material examinado: BRASIL. Mato Grosso do Sul: Rio Negro, Pantanal do Rio Negro, 19³0'12,5"S e $55^{\circ} 36^{\prime} 40,2^{\prime \prime} \mathrm{W}$, sobre colmo de palmeiras, 25-VI2006, V.L.R. Bononi et al. s.n. (SP381529); idem, 25-VI-2006, V.L.R. Bononi et al. s.n. (SP381540).

A espécie ocorre com freqüência nos Estados Unidos da América, sobre madeira de angiospermas, causando podridão branca (Gilbertson \& Ryvarden 1986); citada para México e América do Sul (Montoya et al. 2003). Segundo Moncalvo \& Ryvarden (1997), Ganoderma reniformis Morgan, citado para os Estados do Pará e Santa Catarina por A. C. Batista (Da Silva \& Minter 1995) provavelmente é sinônimo de $G$. lobatum. A espécie tem sido usada com sucesso para a degradação de pentaclorofenol (Gottlieb \& Wright 1999). É encontrada em região de Mata Atlântica no Brasil e vem sendo utilizado para o tratamento e detoxificação de efluentes líquidos (Machado et al. 2005).

Ganoderma lucidum (Curtis) P. Karst., Revue mycol. 3(9): 17. 1991 = Boletus lucidus Curtis, Fl. Londin.: 72. 1781.

Descrições e ilustrações: Gottlieb \& Wright (1999), Nuñez \& Ryvarden (2000).

Material examinado: BRASIL. Mato Grosso do Sul: Rio Negro, Pantanal do Rio Negro, sobre tronco em decomposição, 25-III-2006, A.K.M. de Oliveira \& J.R. Quevedo s.n. (SP381336).
A espécie é cosmopolita, sendo bem conhecida na China onde é utilizada contra tumores (Bernicchia 1990, Gilbertson \& Ryvarden 1986, Guzmán 2003). Gottlieb \& Wright (1999) citam a espécie para a América do Sul. No Brasil, foi citada para Pernambuco, Mato Grosso e Santa Catarina (Fidalgo 1968a, Gibertoni \& Cavalcanti 2003, Loguercio-Leite et al. 2005, Góes Neto \& Baseia 2006).

Ganoderma oerstedii (Fr.) Murrill, Bull. Torrey bot. Club 29: 606. 1907 = Polyporus oerstedii Fr., Nova Acta Soc. Sci. Upsal. Ser. 3: 1. 1851.

Descrição e ilustrações: Ryvarden (2004).

Material examinado: BRASIL. Mato Grosso do Sul: Rio Negro, Pantanal do Rio Negro, sobre colmo da palmeira bacuri ou acuri (Attalea phalerata), II2006, G.B. Soares et al. s.n. (SP381329); idem, sobre madeira em decomposição, 25-III-2006, A.K.M. de Oliveira \& J.R. Quevedo s.n. (SP381914).

Segundo Ryvarden (2004), Ganoderma oerstedii, cuja localidade-tipo é Costa Rica, deve estar amplamente distribuído nas regiões neotropicais. No Brasil, é citada para Santa Catarina e Rio Grande do Sul (Rick 1960, como Ganoderma lobatum var. oerstedii, Loguercio-Leite et al. 2005).

\section{GLOEOPHYLLACEAE}

Gloeophyllum striatum (Sw.) Murrill, Bull. Torrey bot. Club 32: 370. 1905 = Agaricus striatum Sw., Nova Genera Species Plantarum: 148. 1788. (1999).

Descrição e ilustrações: Gugliotta \& Bononi

Material examinado: BRASIL. Mato GRosso do SuL: Rio Negro, Pantanal do Rio Negro, sobre madeira em decomposição, 24-VI-2006, V.L.R. Bononi et al. s.n. (SP381911).

Espécie de distribuição pantropical, ocorrendo principalmente em madeira de angiospermas, ocasionalmente em gimnospermas (Gilbertson \& Ryvarden 1986); amplamente distribuída no Brasil (Gugliotta \& Bononi 1999), foi citado para Mato Grosso por Fidalgo (1968a).

\section{HYMENOCHAETACEAE}

Inonotus patouillardii (Rick) Imazeki, Bulletin of the Tokyo Sci. Mus. 6: 105. 1943 = Polystictus patouillardi Rick, Broteria, ser. bot. 6: 89. 1907.

Descrição e ilustrações: Ryvarden (2004). 
Material examinado: BRASIL. Mato Grosso do Sul: Rio Negro, Pantanal do Rio Negro, sobre madeira em decomposição, 18-II-2006, G.B. Soares et al. s.n. (SP381510).

A espécie é cosmopolita (Ryvarden 2004), e nos Estados Unidos da América, causa podridão branca em Quercus (Gilbertson \& Ryvarden 1986). No Brasil, é citada para Santa Catarina (Drechsler-Santos 2008) e descrita como frequiente no Rio Grande do Sul por Rick $(1928,1961)$ sobre troncos queimados.

Phellinus gilvus (Schwein.) Pat., Essay Taxonomie Hyménomycetes: 82. 1900 三Boletus gilvus Schwein., Schr. naturf. Ges. Leipzig 1: 96. 1822.

Descrições e ilustrações: Soares \& Gugliotta (1998) e Ryvarden (2004).

Material examinado: BRASIL. Mato Grosso do SUL: Rio Negro, Pantanal do Rio Negro, 19³0'36,5'S e $55^{\circ} 37^{\prime} 44,7$ ' W, sobre madeira em decomposição 24VI-2006, V.L.R. Bononi et al. s.n. (SP381530); idem,

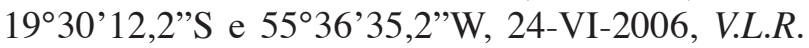
Bononi et al. s.n. (SP381531).

A espécie é cosmopolita (Fonsêca 1999, Ryvarden 2004). Ocorre com freqüência no Brasil, citado para os Estados do Amazonas, Pará, Rondônia, Mato Grosso, Goiás (Fidalgo 1968a, Sousa 1980, Sotão et al. 2002), Rio Grande do Norte, Sergipe, Pernambuco, Alagoas, Paraíba (Gibertoni 2004, Gibertoni et al. 2004, Góes Neto \& Baseia 2006) São Paulo (Jesus 1993, Soares \& Gugliotta 1998, Fonsêca 1999), Rio Grande do Sul (Silveira \& Guerrero 1991).

Phellinus melleoporus (Murrill) Ryvarden, Mycotaxon 23: 177 . 1985 =Fomitoporella melleopora Murrill, N. Amer. Fl. 9: 13. 1907.

Descrição e ilustrações: Ryvarden (2004).

Material examinado: BRASIL. Mato Grosso do Sul: Rio Negro, Pantanal do Rio Negro, sobre pimenteira, em mata ciliar do rio Correntoso, $19^{\circ} 30^{\prime} 43,6^{\prime \prime} \mathrm{S}$ e $55^{\circ} 37^{\prime} 47,8^{\prime \prime} \mathrm{W}, 24-\mathrm{III}-2006$, V.L.R. Bononi s.n. (SP381912).

A espécie ocorre nas Américas, inclusive América do Sul (Gilbertson \& Ryvarden 1987, Ryvarden 2004). Citado para o Brasil para a Paraíba, Rio Grande do Norte e Bahia (Góes Neto et al. 2000, Gibertoni 2004, Gibertoni et al. 2004, Góes Neto \& Baseia 2006).

Phylloporia ribis (Schumach.) Ryvarden, Grundr. Krauterk. 2: 371. 1978 =Boletus ribis Schumach., Enum. pl. 2: 386. 1803.
Descrições e ilustrações: Gilbertson \& Ryvarden (1987), Nuñez \& Ryvarden (2000).

Material examinado: BRASIL. MAto Grosso do Sul: Rio Negro, Pantanal do Rio Negro, $19,49^{\circ} \mathrm{S}$ e $55,62^{\circ} \mathrm{W}$, sobre tronco de angico caído, 25-VI-2006, V.L.R. Bononi et al. 32 (SP).

A espécie é cosmopolita (Bernicchia 1990). No Brasil foi citada para os Estados do Amazonas, Pará, Rondônia, Goiás (Sousa 1980, como Phellinus ribis (Schum. ex Fr.) Quél.) e de São Paulo (Fidalgo \& Fidalgo 1957, Fidalgo et al. 1965, ambos como Phellinus ribis) e, especificamente neste Estado, para a Ilha do Cardoso (Bononi 1979, como Phellinus ribis).

\section{HYPHODERMATACEAE}

Hypochnicium sphaerosporum (Höhn. \& Litsch.) J. Erikss., Symb. bot. upsal. 16: 101. 1958 三 Peniophora sphaerospora Höhn. \& Litsch., Sber. Akad. Wiss. Wien, Math.-naturw. Kl. 115: 1600. 1906.

Descrições e ilustrações: Cunningham (1963), Eriksson \& Ryvarden (1976).

Material examinado: BRASIL. Mato Grosso do Sul: Rio Negro, Pantanal do Rio Negro, sobre madeira em decomposição, 18-VIII-2006, A.K.M. de Oliveira \& J.R. Quevedo s.n. (SP381915).

A espécie apresenta ampla distribuição, sendo citada para o Norte da Europa (Eriksson \& Ryvarden 1976), América do Norte, África do Sul e Nova Zelândia (Cunningham 1963, como Corticium punctulatum Cooke). No Brasil foi citada para São Paulo (Jesus 1993).

Hypochnicium vellereum (Ellis \& Cragin) Parmasto, Conspectus Systematis Corticiacearum: 116. 1968 $\equiv$ Corticium vellereum Ellis \& Cragin, Bulletin of the Washburn Coll. Lab. Nat. Hist. 1: 66. 1885.

Basidioma ressupinado, membranáceo, branco a rosado, 0,1-0,2 $\mathrm{mm}$ esp. Superfície himenial lisa a pulverulenta; margem fibrilosa concolor. Sistema hifálico monomítico; hifas generativas com ansas, parede fina ou pouco espessada, 3-5 um diâm. Basídios tubulares, levemente sinuosos, 50-60 ×6 $\mu \mathrm{m}$, tetraesporados. Basidiósporos globosos, hialinos, parede espessa, cianófilos, 7-8 $\mu \mathrm{m}$ diâm. Conídios presentes, globosos, parede espessada $(2 \mu \mathrm{m})$, intercalares ou terminais, $8-10 \times 6-8 \mu \mathrm{m}$.

Ilustrações: Eriksson \& Ryvarden (1976). 
Material examinado: BRASIL. MAto Grosso DO Sul: Rio Negro, Pantanal do Rio Negro, $19,49^{\circ} \mathrm{S}$ e $55,62^{\circ} \mathrm{W}$, sobre tronco muito podre, $25-\mathrm{VI}-2006$, V.L.R. Bononi et al. s.n. (SP381538).

A espécie ocorre na Dinamarca, Suécia (Eriksson \& Ryvarden 1976) e Itália (Bernicchia 2000), estando na lista de espécies ameaçadas da Dinamarca. Esta é a primeira citação para o Brasil.

\section{LACHNOCLADIACEAE}

Lachnocladium brasiliense (Lév.) Pat., Bull. Torrey bot. Club 29: 572. 1902 E Eriocladus brasiliensis Lév., Suppl. Lich., Paris 5: 108. 1846.

Descrições e ilustrações: Burt (1919), Corner (1970).

Material examinado: BRASIL. Mato Grosso DO Sul: Rio Negro, Pantanal do Rio Negro, sobre restos vegetais muito decompostos, 25-II-2006, A.K.M. de Oliveira \& J.R. Quevedo s.n. (SP381896).

A espécie ocorre nas Américas Central e do Sul; no Brasil, citada para os Estados da Bahia (localidadetipo, Burt 1919), São Paulo e Rio Grande do Sul (Bononi 1980).

Stalpers (1996) considera que esse gênero precisa revisão. Gibertoni et al. (2003) cita Lachocladium weinfurtianum P. Henn. para o nordeste do Brasil, espécie não reconhecida como sinônimo no Index Fungorum.

\section{MARASMIACEAE}

Oudemansiella canarii (Jungh.) Höhn., Akad. Wiss. Wien Math.-Naturw. Kl. 118: 276. 1909 =Agaricus canarii Jungh., Batav. Geroot. Kunst. Wetens. Verh. 17: 82. 1838.

Descrição e ilustrações: Capelari \& Gugliotta (2005).

Material examinado: BRASIL. Mato Grosso do Sul: Rio Negro, Pantanal do Rio Negro, $19^{\circ} 30^{\prime} \mathrm{S}$ e $55^{\circ} 37^{\prime} \mathrm{W}$, sobre tronco em decomposição, 24-VI2006, V.L.R. Bononi et al. s.n. (SP381322).

Espécie de distribuição Pantropical (Pegler 1997), citada para Venezuela (Dennis 1970), Argentina (Singer \& Digilio 1952) e Brasil, nos Estados do Amazonas, Rondônia, Pernambuco, Rio de Janeiro, São Paulo e Rio Grande do Sul (Putzke \& Pereira 1988, Capelari 1989, Capelari \& Gugliotta 2005).

\section{MERULIACEAE}

Gloeoporus thelephoroides (Hook.) G. Cunn., Bull. N. Z. Dept. Sci. Industr. Res. 164: 111. $1965 \equiv$ Boletus thelephoroides Hook., Syn. pl. 1: 10. 1822.

Descrições e ilustrações: Gilbertson \& Ryvarden (1986), Corner (1989).

Material examinado: BRASIL. Mato GRosso do Sul: Rio Negro, Pantanal do Rio Negro, 19³2'10'7'S e $56^{\circ} 36^{\prime} 33,6^{\prime \prime} \mathrm{W}$, sobre tronco em decomposição, 24VI-2006, V.L.R. Bononi et al. s.n. (SP381546); idem, 18-VIII-2006, A.K.M. de Oliveira \& J.R. Quevedo s.n. (SP381894).

A espécie apresenta distribuição pantropical (Gilbertson \& Ryvarden 1986); no Brasil, é citada para Amazonas, Mato Grosso e Santa Catarina (Fidalgo 1968a, Corner 1989, Drechsler-Santos 2008).

Mycoaciella bispora (Stalpers) J. Erikss. \& Ryvarden, Corticiaceae of North Europe 5: $901.1978 \equiv$ Resinicium bisporum Stalpers, Persoonia 9: 145. 1976 = Phlebia bispora (Stalpers) Nakasone, Mycotaxon 84: 481. 2002.

Basidioma ressupinado, creme a ocráceo, fino, até $2 \mathrm{~mm}$ esp. Superfície himenial hidnóide, dentes até $2 \mathrm{~mm}$ compr.; margem concolor à superfície. Sistema hifálico dimítico; hifas generativas com ansas, parede fina, 2-3 um diâm, hifas esqueléticas com parede espessa, 2-3 $\mu \mathrm{m}$ diâm. Presença de cristais no contexto. Cistídios presentes, cilíndricos, com ápice dilatado, arredondado e coberto por material resinoso amarelado, 20-30 × 4-5 $\mu \mathrm{m}$. Basídios clavados, 15-20 $\times$ 3-4 $\mu \mathrm{m}$, tetraesporados. Basidiósporos elipsóides a cilíndricos, parede lisa, 4-5 × 2,5-3 $\mu \mathrm{m}$.

Ilustrações: Eriksson et al. (1978), Nakasone (2002).

Material examinado: BRASIL. Mato Grosso do SuL: Rio Negro, Pantanal do Rio Negro, 19³0'34,8'S e $55^{\circ} 37^{\prime} 45,9^{\prime \prime} \mathrm{W}$, sobre tronco em decomposição 24-VI2006, V.L.R. Bononi et al. s.n. (SP381541).

A espécie ocorre nos Estados Unidos da América (Nakasone 2002) e Europa (Eriksson et al. 1978, Nakasone 2002). Esta é a primeira citação de ocorrência no Brasil.

\section{PLEUROTACEAE}

Pleurotus agaves Dennis, Kew Bull., Addit. Ser. 3: 466. 1970. 
Basidioma pileado, flabeliforme, lateralmente subestipitado, carnoso, branco, $2 \times 2 \mathrm{~cm}$. Superfície himenial lamelar, branca. Queilocistídios cilíndricos a ventricosos, parede fina, 25-30 ×8-10 $\mu \mathrm{m}$. Pleurocístidios ausentes. Sistema hifálico monomítico; hifas generativas com ansas, parede fina, 3-4 $\mu$ m diâm. Basídios clavados, 15-20 × 5-8 um, tetraesporados. Basidiósporos oblongos, hialinos, parede lisa, 7-8 × 3-3,5 um.

Ilustrações: Dennis (1970).

Material examinado: BRASIL. MAto Grosso DO Sul: Rio Negro, Pantanal do Rio Negro, 30-IX-2006, A.K.M. de Oliveira s.n. (SP381899).

Espécie conhecida para a Venezuela (Dennis 1970). Esta é a primeira citação para o Brasil.

\section{POLYPORACEAE}

Coriolopsis polyzona (Pers.) Ryvarden, Norw. Jl. Bot. 19: 230. 1972 三 Polyporus polyzonus Pers. in Gaudicheau, Voyage. Autour du. Monde: 170. 1827.

Descrições e ilustrações: Ryvarden \& Johansen (1980), Fonsêca (1999), Guzmán (2003).

Material examinado: BRASIL. Mato Grosso do Sul: Rio Negro, Pantanal do Rio Negro, 18-VIII2006, A.K.M. de Oliveira \& J.R. Quevedo s.n. (SP381655).

A espécie apresenta distribuição pantropical; citada no Brasil para os Estados de Pernambuco (Cavalcanti 1979, como Coriolus occidentalis (Sw.:Fr.) Kreisel), Bahia, Rio de Janeiro, São Paulo, Paraná e Santa Catarina (Fonsêca 1999, Gerber \& Loguercio-Leite 2000).

Hexagonia hydnoides (Sw.) M. Fidalgo, Mem. N. Y. bot. Gdn. 17: 64. 1968 = Boletus hydnoides Sw., Fl. Ind. Occid. 3: 1942. 1806.

Descrições e ilustrações: Fidalgo (1968b), Gugliotta \& Bononi (1999).

Material examinado: BRASIL. Mato Grosso Do Sul: Rio Negro, Pantanal do Rio Negro, I-2006, G.B. Soares et al. s.n. (SP381323); idem, I-2006, G.B. Soares et al. s.n. (SP381324); idem, 24-III-2006, V.L.R. Bononi s.n. (SP381325); idem, 19³0’34,9'S

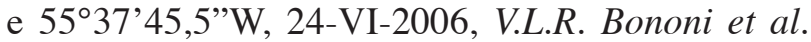
s.n. (SP381916); idem, 19³0'9, 8'S e 55³6'42,7''W, sobre madeira em decomposição, 25-VI-2006, V.L.R. Bononi et al. s.n. (SP381527); idem, 19³0'11,4”S e
55³6'9,8’W, 25-VI-2006, V.L.R. Bononi et al. s.n. (SP381528).

Espécie de distribuição pantropical e freqüente em áreas semi-áridas e savanas. Está amplamente distribuída no Brasil, citada para os Estados do Amazonas, Roraima, Rondônia, Maranhão, em manguezais, Pará, Alagoas, Rio Grande do Norte, Paraíba, Pernambuco, Sergipe, Bahia, Maranhão, Goiás, Mato Grosso, Minas Gerais, Rio de Janeiro, Espírito Santo, São Paulo, Paraná, Santa Catarina e Rio Grande do Sul (Fidalgo 1968b, Capelari \& Maziero 1988, Vinha 1988, Loguercio-Leite \& Wright 1991, Jesus 1996, Gugliotta \& Bononi 1999, Ryvarden \& Meijer 2002, Sotão et al. 2002, Sotão et al. 2003, Xavier-Santos 2003, Gibertoni 2004, Gibertoni et al. 2004, Góes Neto \& Baseia 2006, Meijer 2006).

Hexagonia variegata Berk., Ann. Mag. nat. Hist., Ser. 2, 9: 196. 1852.

Descrições e ilustrações: Fidalgo (1968b), Gugliotta \& Bononi(1999), como Hexagonia papyracea Berk., atualmente colocado em sinonímia.

Material examinado: BRASIL. MAto GRosso do SuL: Rio Negro, Pantanal do Rio Negro, sobre madeira em decomposição, II-2006, G.B. Soares et al. s.n. (SP381320); idem, madeira em decomposição, 25III-2006, A.K.M. de Oliveira \& J.R. Quevedo s.n. (SP381545); idem, 19³0'43,6”'S e 55'37'47,8'W, 24III-2006, V.L.R. Bononi et al. s.n. (SP381917); idem, 18-VIII-2006, A.K.M. de Oliveira \& J.R. Quevedo s.n. (SP381918).

A espécie apresenta distribuição pantropical e está amplamente distribuída no Brasil, citada como Hexagonia papyracea, para Amazonas, Alagoas, Rio Grande do Norte, Paraíba, Pernambuco, Sergipe, Bahia, Goiás, Minas Gerais, Rio de Janeiro, São Paulo, Paraná, Santa Catarina e Rio Grande do Sul (Fidalgo 1968b, Gugliotta \& Bononi 1999, Ryvarden \& Meijer 2002, Xavier-Santos 2003, Gibertoni 2004, Gibertoni et al. 2004, Góes Neto \& Baseia 2006, Meijer 2006).

Lentinus concavus (Berk.) Corner, Beih. Nova Hedwigia 69: 30. 1981 = Panus concavus Berk., Ann. Mag. nat. Hist., Ser. 2, 9: 194. 1852.

Basidioma pileado estipitado Píleo fino e firme, convexo-depresso, infundibuliforme. Superfície abhimenial lisa, creme a castanha. Superfície himenial lamelar, lamelas decurrentes, creme, até $2 \mathrm{~mm}$ espessas, com lamélulas; margem inteira. Estipe delgado, 
cilíndrico, ligeiramente excêntrico, sólido, branco, estriado, glabro, $4 \times 2 \mathrm{~mm}$. Véu parcial presente, submembranoso. Contexto fino, branco dimítico; hifas generativas com ansas, pouco ramificadas, parede fina, 2-5 $\mu \mathrm{m}$ diâm.; hifas esqueléticas pouco ramificadas, parede pouco espessada, 4-10 um diâm. Queilocistidios clavóides, hialinos, parede fina, 15$25 \times 3-5 \mu \mathrm{m}$. Basídios clavados, 14-15 × 4-5 $\mu \mathrm{m}$ tetraesporados, esterigmas curtos. Basidiósporos cilíndricos, hialinos, 6-7 × 1,5-2,5 um.

Ilustrações: Pegler (1983b).

Material examinado: BRASIL. MAto Grosso do SuL: Rio Negro, Pantanal do Rio Negro, 26-VI-2006, V.L.R. Bononi et al. s.n. (SP381327)

Espécie de ocorrência na América tropical, já citada para Cuba, Peru, Republica Dominicana, Trinidad e Venezuela (Pegler 1983b). Esta é a primeira citação específica para o Brasil.

Lentinus crinitus (L.) Fr., Nov. Symb Myc.: 34. 1825 三Agaricus crinitus L., Sp. pl., Edn. 2, 2: 1644. 1763.

Descrições e ilustrações: Pegler (1983b), Fonsêca (1999).

Material examinado: BRASIL. Mato Grosso do Sul: Rio Negro, Pantanal do Rio Negro, 19³0'46,8'S e 55³7'47,6”'W, 24-III-2006, V.L.R. Bononi s.n. (SP 381920); idem, 18-VIII-2006, A.K.M. de Oliveira \& J.R. Quevedo s.n. (SP381919).

A espécie apresenta distribuição pantropical, encontrada do México até a Argentina. Possui ampla distribuição no Brasil (Fidalgo 1968a, Capelari \& Maziero 1988, Pegler 1997, Fonsêca 1999, Sotão et al. 2002, Gibertoni 2004, Gibertoni et al. 2004, Góes Neto \& Baseia 2006).

Lentinus swartzii Berk., J. Bot., London 2: 632. 1843.

Descrição e ilustrações: Pegler (1983b).

Material examinado: BRASIL. Mato Grosso do Sul: Rio Negro, Pantanal do Rio Negro, 30-IX-2006, A.K.M. de Oliveira s.n. (SP381921).

A espécie ocorre na América Central e América do Sul (Dennis 1970, como L. nicoatianus Berk., Pegler 1983b, 1997). No Brasil foi citada para os Estados do Amazonas, Bahia, Mato Grosso, Minas Gerais, Rio de Janeiro e São Paulo (Pegler 1983b, 1997).

Nigroporus macroporus Ryvarden \& Iturr., Mycologia 95: 1070. 2003.
Basidioma anual a perene, pileado séssil a subestipitado, $5 \times 8 \mathrm{~cm}$, até $1 \mathrm{~cm}$ esp., coriáceo a rígido. Superfície abhimenial glabra, castanhoescura a preta, sulcada. Superfície himenial poróide, castanho-escura, 1-2 poros/mm; tubos 5-7 mm compr. Contexto castanho-escuro com fina cutícula ou zona preta próxima ao substrato. Sistema hifálico dimítico; hifas generativas com ansas, hialinas 1,5-3 um diâm.; hifas esqueléticas castanho-claras, parede espessada a sólida, 3-6 um diâm. Basídios clavados, 5-7 × 10$15 \mu \mathrm{m}$, tetraesporados. Basidiósporos cilíndricos, hialinos, parede fina, lisa, 5-6 × 1,8-2 $\mu \mathrm{m}$. Cistídios presentes, filiformes, 2-6 $\mu \mathrm{m}$ diâm. e até 2 vezes a altura dos basídios.

Ilustrações: Ryvarden \& Iturriaga (2003).

Material examinado: BRASIL. Mato Grosso do SuL: Rio Negro, Pantanal do Rio Negro, sobre tronco em decomposição, 25-III-2006, A.K.M. Oliveira \& J.R. Quevedo s.n. (SP381520).

Espécie citada para a Venezuela (Ryvarden \& Iturriaga 2003). Esta é a segunda anotação de ocorrência da espécie e a primeira para o Brasil.

Perenniporia martii (Berk.) Ryvarden, Norw. Jl. bot. 19: $143.1972 \equiv$ Polyporus martius Berk., Hooker's J. Bot. 8: 198. 1856.

Descrições e ilustrações: Teixeira (1948), Ryvarden \& Johansen (1980).

Material examinado: BRASIL. Mato Grosso do SuL: Rio Negro, Pantanal do Rio Negro, sobre tronco em decomposição, 23-III-2006, A.K.M. de Oliveira \& J.R. Quevedo s.n. (SP381521).

A espécie apresenta distribuição pantropical (Ryvarden \& Johansen 1980) e no Brasil foi citada para Paraíba, Rio Grande do Norte, Pernambuco, Bahia, São Paulo, Paraná, Santa Catarina e Rio Grande do Sul (Teixeira 1948, como Fomitopsis honodermus (Mont.) Singer, Gonçalves \& Loguercio-Leite 2001, Gibertoni 2004).

Polyporus tenuiculus (P. Beauv.) Fr., Syst. mycol. 1: 344. 1821 EFavolus tenuiculus $\mathrm{P}$. Beauv., Fl. Oware 1: 74. 1806.

Descrições e ilustrações: Nuñez \& Ryvarden (1995), Silveira \& Wright (2005).

Material examinado: BRASIL. Mato Grosso do Sul: Rio Negro, Pantanal do Rio Negro, sobre madeira,

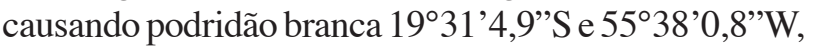
24-III-2006, V.L.R. Bononi s.n. (SP381922). 
A espécie, que apresenta distribuição pantropical (Fonsêca 1999), é comum no Brasil, citada para os Estados do Amazonas, Roraima, Pará, Rondônia, Bahia, Alagoas, Paraíba, Pernambuco, Sergipe, Mato Grosso, Rio de Janeiro, São Paulo, Paraná, Santa Catarina, Rio Grande do Sul (Singer 1961, como Polyporus dermoporus Pers., Fidalgo 1974, Corner 1984, como Polyporus brasiliensis (Fr.) Corner, Capelari \& Maziero 1988, como F. brasiliensis, Sotão et al. 1997, 2002, Fonsêca 1999, Góes Neto 1999, Gugliotta \& Bononi 1999, Gonçalves \& LoguercioLeite 2001, Ryvarden \& Meijer 2002, Gibertoni \& Cavalcanti 2003, Góes Neto et al. 2003, Gibertoni et al. 2004, Silveira \& Wright 2005, Góes Neto \& Baseia 2006, Silva \& Gibertoni 2006, Xavier-Santos 2003).

Pycnoporus sanguineus (L.) Murrill, Bull. Torrey bot. Club 31: 421.1904 =Boletus sanguineus L., Sp. pl., Edn 2: 1646. 1763.

Descrições e ilustrações: Gilbertson \& Ryvarden (1987), Gugliotta \& Bononi (1999).

Material examinado: BRASIL. Mato Grosso do Sul: Rio Negro, Pantanal do Rio Negro, $19^{\circ} 30^{\prime} 35,8^{\prime \prime} \mathrm{S}$ e 55³7'45,1”W, 24-VI-2006, V.L.R. Bononi et al. s.n. (SP 381923); idem, 18-VIII-2006, A.K.M. Oliveira \& J.R. Quevedo s.n. (SP381924).

A espécie apresenta distribuição pantropical (Ryvarden \& Johansen 1980) e está amplamente distribuída no Brasil, citada para o Pará, Alagoas, Paraíba, Pernambuco, Bahia, São Paulo, Paraná, Santa Catarina e Rio Grande do Sul (Loguercio-Leite \& Wright 1991, Silveira \& Guerrero 1991, Gugliotta \& Bononi 1999, Sotão et al. 2002, Xavier-Santos 2003, Gibertoni et al. 2004, Góes Neto \& Baseia 2006, Meijer 2006). Tem sido utilizada na produção de enzimas como a alfa-amilase e na degradação de corantes utilizados na indústria têxtil (Bononi \& Grandi 1998).

Trametes cubensis (Mont.) Sacc., Syll. fung. 9: 198. 1891 = Polyporus cubensis Mont., Annls. Sci. Nat., Bot. sér. 2, 8: 364. 1837.

Descrições e ilustrações: Gilbertson \& Ryvarden (1987), Gugliotta \& Bononi (1999).

Material examinado: BRASIL. Mato Grosso do Sul: Rio Negro, Pantanal do Rio Negro, 19³0’36”S e 55³7'46,5”'W, 24-VI-2006, V.L.R. Bononi et al. s.n. (SP381925); idem, $19,49^{\circ} \mathrm{S}$ e $55,62^{\circ} \mathrm{W}$, sobre madeira em decomposição, 25-VI-2006, V.L.R. Bononi et al. s.n. (SP381537).
Espécie com distribuição neotropical (Gilbertson \& Ryvarden 1987). No Brasil, foi citada para os Estados do Amazonas, Pernambuco, Bahia, São Paulo, Paraná sobre Pinus sp., Santa Catarina e Rio Grande do Sul (Loguercio-Leite \& Wright 1991, Gugliotta \& Bononi 1999, Ryvarden \& Meijer 2002, Gibertoni et al. 2004, Góes Neto \& Baseia 2006).

Trametes subectypus (Murrill) Gilbn. \& Ryvarden, N. Amer. Polyp. 2: 758. 1987 ECoriolus subectypus Murrill, N. Amer. Fl. 9: 22. 1907.

Basidioma anual, pileado séssil a subestipitado, aplanado, coriáceo a rígido, $8 \times 8 \times 0,5 \mathrm{~cm}$. Superfície abhimenial branca a creme, azonada ou fracamente zonada, com zonas velutinosas e glabras. Superfície himenial poróide, branca a creme, poros angulares, 5-7 poros $/ \mathrm{mm}$; tubos de $4 \mathrm{~mm}$ compr. Contexto branco, fino, $1 \mathrm{~mm}$ espesso. Sistema hifálico trimítico, hifa generativas com ansas, hialinas, 2-4 um diâm.; hifas esqueléticas abundantes, parede espessada, 2-8 $\mu \mathrm{m}$ diâm.; hifas conectivas tortuosas, muito ramificadas, sólidas, 2-5 um diâm. Basídios clavados, 10-12 × 4-5 $\mu \mathrm{m}$, tetraesporados. Basidiósporos cilíndricos, hialinos, parede lisa, 3,5-5 × 1-2 $\mu \mathrm{m}$.

Ilustrações: Gilbertson \& Ryvarden (1987).

Material examinado: BRASIL. MAto GROSSO Do SUL: Rio Negro, Pantanal do Rio Negro, 19³0'12,3'S e 55³6'8, ''W, sobre tronco em decomposição, 24-VI2006, V.L.R. Bononi et al. s.n. (SP381542).

Esta espécie ocorre nos Estados Unidos da América causando podridão branca em angiospermas (Gilbertson \& Ryvarden 1987). Esta é a primeira citação para o Brasil.

Trametes villosa (Sw.) Kreisel, Monografia Ciencias, Univ. Habana, Ser. 4, 16: 84. 1971 = Boletus villosus Sw., Fl. Ind. Occid. 3: 1923. 1806 = Polyporus villosus Fr., Syst. Mycol. 1: 344. 1821.

Descrições e ilustrações: Gilbertson \& Ryvarden (1987), Gugliotta \& Bononi (1999).

Material examinado: BRASIL. MAto Grosso do Sul: Rio Negro, Pantanal do Rio Negro, $19,49^{\circ} \mathrm{S}$ e $55,62^{\circ} \mathrm{W}$, sobre madeira em decomposição, 25-VI2006, V.L.R. Bononi et al. s.n. (SP381547).

Espécie de distribuição neotropical e comum no Brasil. Citada para os Estados de Roraima Amapá, Bahia, São Paulo, Paraná, Santa Catarina e Rio Grande do Sul (Sotão et al. 1991, Jesus 1996, Fonsêca 1999, Gibertoni et al. 2004, Góes Neto \& Baseia 2006, Meijer 2006). 
Trichaptum perrottetii (Lév.) Ryvarden, Norw. Jl. Bot. 19: 237. 1972 = Trametes perrottetii Lév., Annls. Sci. Nat., Bot. sér.3, 2: 195. 1844.

Descrição e ilustrações: Gilbertson \& Ryvarden (1987).

Material examinado: BRASIL. Mato Grosso do SuL: Rio Negro, Pantanal do Rio Negro, sobre madeira em decomposição, 25-III-2006, A.K.M. de Oliveira \& J.R. Quevedo s.n. (SP381321).

A espécie já foi citada para as Américas, dos Estados Unidos da América (Flórida) até a Argentina, incluindo o Brasil, Pernambuco (Gilbertson \& Ryvarden 1987, Gibertoni et al. 2004).

Tyromyces fumidiceps G.F. Atk., Annls. mycol. 6: 61. 1908.

Descrições e ilustrações: Gilbertson \& Ryvarden (1987), Fonsêca (1999).

Material examinado: BRASIL. Mato Grosso do SuL: Rio Negro, Pantanal do Rio Negro, sobre tronco em decomposição, 24-VI-2006, V.L.R. Bononi et al. s.n. (SP381334).

A espécie é rara na Europa, citada para os Estados Unidos da América, Canadá e, no Brasil, para o Estado de São Paulo, na Reserva Biológica de Paranapiacaba (Fonsêca 1999, como Tyromyces pseudolacteus Murrill, hoje colocado em sinonímia).

\section{PSATHYRELLACEAE}

Psathyrella murrillii A.H. Sm., Mem. N. Y. bot. Gdn. 24: 287. 1972.

Descrições e ilustrações: Smith (1972), Pegler (1983a).

Material examinado: BRASIL. Mato Grosso Do Sul: Rio Negro, Pantanal do Rio Negro, no solo, $19^{\circ} 30^{\prime} 34,8^{\prime}$ 'S e $55^{\circ} 37^{\prime} 45,9^{\prime} \mathrm{W}, 24-\mathrm{III}-2006$, V.L.R. Bononi et al. s.n. (SP381900).

A espécie ocorre na América tropical (Pegler 1997), sendo comum em Martinica e Cuba (Pegler 1983a). No Brasil, citada para o Estado de São Paulo (Pegler 1997).

\section{SCHIZOPHYLLACEAE}

Schizophyllum commune Fr., Observ. mycol. 1: 103. 1815
Descrição e ilustrações: Silva \& Gibertoni (2006).

Material examinado: BRASIL. MAto Grosso do Sul: Rio Negro, Pantanal do Rio Negro, 19³0'12'S e 55³6'40”'W, 24-III-2006, V.L.R. Bononi et al. s.n. (SP381902); idem, 19³0'33,9”'S e 55³7'45”W, 24III-2006, V.L.R. Bononi et al. s.n. (SP381901).

A espécie é cosmopolita e comum no Brasil sobre cercas e pontes de madeira (Guerrero \& Homrich 1983, Sotão et al. 1997, 2003, Fonsêca 1999, Gibertoni \& Cavalcanti 2003, Xavier-Santos 2003, Meijer 2006, Góes Neto \& Baseia 2006, Silva \& Gibertoni 2006).

\section{SCHIZOPORACEAE}

Echinoporia aculeifera (Berk. \& M.A. Curtis) Ryvarden, Mycotaxon 20: 330. 1984 三 Trametes aculeifera Berk. \& M.A. Curtis, J. Linn. Soc., Bot.10: 319. 1868.

Descrições e ilustrações: Ryvarden (1984), Silveira \& Guerrero (1991).

Material examinado: BRASIL. MAto Grosso do SuL: Rio Negro, Pantanal do Rio Negro, $19^{\circ} 30^{\prime} 10,1^{\prime \prime}$ e 55 36'41,5'W, sobre madeira em decomposição, 26VI-2006, V.L.R. Bononi et al. s.n. (SP381536).

Espécie de distribuição neotropical, ocorre da Flórida ao Brasil (Gilbertson \& Ryvarden 1986). No Brasil, citada para os Estados da Bahia (Góes Neto 1999), São Paulo (Fonsêca 1999) e Rio Grande do Sul (Silveira \& Guerrero 1991).

\section{SISTOTREMATACEAE}

Trechispora regularis (Murrill) Liberta, Can. J. Bot. 51: 1878. 1973 = Poria regularis Murrill, Mycologia 12: 87. 1920.

Descrições e ilustrações: Gilbertson \& Ryvarden (1986), Silveira \& Guerrero (1991).

Material examinado: BRASIL. Mato Grosso do Sul: Rio Negro, Pantanal do Rio Negro, $19^{\circ} 49^{\prime}$ S e $55^{\circ}$ $62^{\prime} \mathrm{W}$, sobre tronco queimado, 25-VI-2006, V.L.R. Bononi et al. s.n. (SP381539).

A espécie ocorre em zonas temperadas e tropicais, citada para África, Estados Unidos da América, Jamaica, Costa Rica, Antilhas, Guiana Francesa, Brasil, no Rio Grande do Sul, Argentina, e Paraguai (Silveira \& Guerrero 1991). 


\section{STEREACEAE}

Stereum ostrea (Blume \& T. Nees) Fr., Epicr. Syst. mycol.: 547. 1838 = Thelephora ostrea Blume \& T. Nees, Nova Acta Phys.-Med. Acad. Caes .Leop.Carol. Nat. Cur. 13: 13. 1826.

Descrições e ilustrações: Burt (1920), Teixeira (1945), Fonsêca (1999, como Stereum australe Lloyd, considerada sinônimo).

Material examinado: BRASIL. Mato Grosso do Sul: Rio Negro, Pantanal do Rio Negro, sobre madeira em decomposição, II-2006, G.B. Soares et al. s.n. (SP381543).

Espécie de distribuição pantropical e citada para Trinidad e Tobago, Guiana, Colômbia e Venezuela (Dennis 1970). Ocorre no Brasil, sendo citado para os Estados de Alagoas, Rio Grande do Norte, Paraíba, Sergipe, Pernambuco, Bahia, Rio de Janeiro, São Paulo e Paraná (Teixeira 1945, Jesus 1993, Fonsêca 1999, Gibertoni \& Cavalcanti 2003, Gibertoni et al. 2006, Góes Neto \& Baseia 2006, Meijer 2006). Registros recentes na internet apontam sua presença na Nova Zelândia e China onde ela é utilizada na alimentação (http://www.nzfungi.landcareserch.co.nz, acesso em 12.12.2006 e http://www.engine.cqvip.com, acesso em 12.12.2006). Não foram encontradas publicações confirmando a informação.

\section{STROPHARIACEAE}

Hypholoma trinitense (Dennis) Pegler, Kew Bull., Addit. Ser. 9: 509. 1983 = Pholiota trinitensis Dennis, Kew Bull., Addit. Ser. 3: 467. 1970.

Descrição e ilustração: Pegler (1983a).

Material examinado: BRASIL. MAto GRosso do SuL: Rio Negro, Pantanal do Rio Negro, 19³0’34,8”S e $55^{\circ} 37^{\prime} 43,9^{\prime \prime} \mathrm{W}$, em solo muito arenoso, nas margens do Rio Correntoso, 24-VI-2006, V.L.R. Bononi et al. s.n. (SP381903).

Espécie de ocorrência na América tropical, citada para Martinica, Trinidad e no Brasil, para o Estado de São Paulo (Pegler 1997). Segundo Pegler (1983a), Nematoloma amazonicum Singer, descrito para o Brasil, é sinônimo de Hypholoma trinitense.

Pholiota polychroa (Berk.) A.H. Sm. \& H.J. Brodie, Bot. Gaz. 96: 533. 1935 = Agaricus polychrous Berk., J. Bot., London 6: 313. 1847.

Basidioma pileado estipitado; píleo carnoso, convexo com largo umbo, castanho-alaranjada, glabro, víscido, $5 \mathrm{~cm}$ diâm. Superfície himenial lamelar, lamelas aderidas ao estipe, ligeiramente decurrentes, com lamélulas ocre. Estipe cilíndrico, sólido, $4 \times$ $0,6 \mathrm{~cm}$, fibroso, superfície castanho-amarelada, mais escura na base. Véu parcial flocoso, efêmero. Trama da lamela regular. Sistema hifálico monomítico, hifas generativas com ansas, hialinas, infladas, 5-20 um diâm. Queilocístidios fusóides com ápice obtuso, hialinos, parede fina, 25-40 × 10-20 $\mu \mathrm{m}$. Pleurocístidios fusóideventricosos, pedicelados, com conteúdo refratório castanho-amarelado, parede fina, 45-60 × 10-15 $\mu \mathrm{m}$. Basídios clavados, 20-22 × 6-7 $\mu \mathrm{m}$, tetraesporados. Basidiósporos elipsóides a amigdaliformes, ócre, parede espessa, lisa, com poro de germinação.

Ilustrações: Pegler (1983a).

Material examinado: BRASIL. Mato Grosso do Sul: Rio Negro, Pantanal do Rio Negro, $19^{\circ} 31^{\prime} 0,4$ 'S e $55^{\circ} 38^{\prime} 0,5^{\prime}$ 'W, sobre madeira em decomposição, nas margens do rio Correntoso, 24-VI-2006, V.L.R. Bononi et al. s.n. (SP381904).

Pegler (1983a) relatou a ocorrência da espécie em Ohio, nos Estados Unidos da América e em Martinica, na América Central. Esta é a primeira citação para o Brasil.

Psilocybe venezuelana Dennis, Kew Bull. 15: 137. 1961.

Descrições e ilustrações: Guzmán (1983), Pegler (1983a).

Material examinado: BRASIL. Mato Grosso do Sul: Rio Negro, Pantanal do Rio Negro, sobre serapilheira, 30-IX-2006, A.K.M. de Oliveira s.n. (SP381905).

A espécie ocorre na América tropical (Pegler 1997), sendo citada para Martinica, Guadalupe, Venezuela (Dennis 1970) e no Brasil, para o Estado de São Paulo (Pegler 1997).

Psylocybe zapotecorum Heim emend. Guzmán var. ramulosum Guzmán \& Bononi, Mycotaxon 19: 346. 1984.

Descrição e ilustrações: Guzmán et al. (1984).

Material examinado: BRASIL. Mato Grosso Do Sul: Rio Negro, Pantanal do Rio Negro, às margens de lagoa, 30-IX-2006, A.K.M. de Oliveira s.n. (SP381906).

Esta variedade foi descrita para o Estado de São Paulo (Guzmán et al. 1984), sendo o Pantanal a segunda localidade. 


\section{THELEPHORACEAE}

Thelephora griseozonata Cooke, Grevillea 19: 104. 1891.

Descrições e ilustrações: Cunningham (1963), Corner (1968).

Material examinado: BRASIL. Mato GRosso do SuL: Rio Negro, Pantanal do Rio Negro, em solo arenoso, 18-VIII-2006, A.K.M. de Oliveira \& J.R. Quevedo s.n. (SP381926).

Espécie de ocorrência na América do Norte e Nova Zelândia (Cunningham 1963, Corner 1968). No Brasil, foi encontrada no Parque Estadual da Ilha do Cardoso no Estado de São Paulo (Bononi 1979).

\section{TRICHOLOMATACEAE}

Collybia bakeri Dennis, Kew Bull., Addit. Ser. 3: 465. 1970.

Basidioma pileado estipitado; píleo campanulado a convexo, com umbo, 1,5-4 cm diâm. Superfície abhimenial canela a castanho-rosada, higrófana, glabra, sulcada. Superfície himenial lamelar, lamelas adnexo-adnatas, esbranquiçadas a creme, com lamélulas de 2 comprimentos. Estipe cilíndrico, 1,5-7 $\times 1-4 \mathrm{~cm}$, micélio basal, liso, concolor à superfície abhimenial a mais escuro. Contexto castanho, até 1 $\mathrm{mm}$ espesso. Trama da lamela regular; hifas hialinas, 4-8 um diâm. Queilocístidios cilíndricos, sinuosos, hialinos,parede fina, 22-35 × 2-4 $\mu \mathrm{m}$. Pleurocístidios ausentes. Basídios clavados, 16-18 × 4-5 $\mu \mathrm{m}$, tetraesporados. Basidiósporos elipsóides, hialinos, parede fina, geralmente unigutulado.

Ilustrações: Pegler (1983a).

Material examinado: BRASIL. Mato Grosso DO Sul: Rio Negro, Pantanal do Rio Negro, em solo, vários basidiomas agrupados, nas margens do Rio

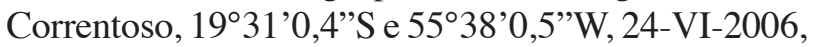
V.L.R. Bononi s.n. (SP381907).

Espécie com referência para Martinica e Trinidad (Dennis 1970). Esta é a primeira citação da espécie para o Brasil.

Collybia neotropica Singer, Sydowia 15: 54. 1961.

Descrição e ilustração: Pegler (1983a).

Material examinado: BRASIL. MATo GRosso do SuL: Rio Negro, Pantanal do Rio Negro, sobre madeira de leira, 19³1,4'6"'S e 55³8'0,9'W, 24-III-2006, V.L.R. Bononi s.n. (SP381908).
A espécie ocorre na América tropical e América do Sul (Pegler 1997), citado para Martinica e Trinidad (Pegler 1983a) sobre folhas de coqueiros. No Brasil, relatada para o Estado de São Paulo (Pegler 1997).

Mycena inclinata (Fr.) Quél., Champignons Jura Vosges: 105. $1872 \equiv$ Agaricus inclinatus Fr., Epicr. Syst. mycol.: 107. 1838.

Descrição: Rick (1961), Smith (1947).

Material examinado: BRASIL. MATO GRosso DO Sul: Rio Negro, Pantanal do Rio Negro, 19³1'4,6”'S

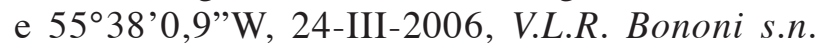
(SP381909).

Espécie com referência para Europa e Estados Unidos da América (Smith 1947, Robich 2006). No Brasil, citado para o Estado do Rio Grande do Sul e Paraná (Rick 1961, Maas-Geesteranus \& Meijer 1995).

Mycena parabolica (Fr.) Quél., Champignons Jura Vosges: 242. 1872 = Agaricus parabolicus Fr., Epicr. Syst. mycol.: 107. 1838.

Basidioma pileado estipitado; píleo cônicocampanulado, às vezes com pequeno umbo no centro, 1,5-2,5 cm diâm. Superfície abhimenial castanhofusca-acinzentada a quase preta, higrófana, sulcada, glabra. Lamelas adnatas, decurrentes, acinzentadas, com lamélulas de 2 comprimentos; margem irregular. Estipe cilíndrico, sólido a oco, concolor à superfície abhimenial a mais claro, glabro, com ápice fibrilosopruinoso, 2,5-4 × 0,2-0,3 cm. Contexto fino, cinzaescuro; hifas hialinas, com ansas, 7-17 $\mu \mathrm{m}$ diâm. Trama da lamela regular. Queilocistídios clavados a piriformes, hialinos, 15-30 × 7-15 $\mu$ m. Pleurocístidios ausentes. Basídios clavados, 15-20 × 6-8 $\mu \mathrm{m}$, tetraesporados. Basidiósporos ovóides a cilíndricos, hialinos, amilóides, parede fina, 8-10 × 5-7 $\mu \mathrm{m}$.

Ilustrações: Dennis (1951).

Material examinado: BRASIL. Mato GRosso do SuL: Rio Negro, Pantanal do Rio Negro, sobre serapilheira, II-2006, G.B. Soares et al. s.n. (SP381330).

A espécie é cosmopolita, citada para Venezuela (Dennis 1970) e ocorrendo em terras úmidas, na região dos lagos americanos de Seattle, no Estado de Washington, Estados Unidos da América, em ambiente alagado como o pantanal (Bessette et al. 1996). Esta é a primeira citação para o Brasil.

Nothopanus hygrophanus (Mont.) Singer, Kew Bull. 23: 247. 1949 Panus hygrophanus Mont., Annls. Sci. Nat., Bot. sér. 4, 1: 122. 1854. 
Basidioma pileado estipitado; píleo reniforme a flabeliforme, 1-3,5 cm diâm. Superfície abhimenial esbranquiçada, com manchas castanho-vináceas próximas à base, higrófana, fibrilosa. Lamelas decurrentes,concolores à superfície abhimenial, com lamélulas de 2 comprimentos. Estipe excêntrico, robusto, com manchas marrom-avermelhadas na base 1-10 × 1-6 mm. Contexto fino, branco; hifas hialinas, com ansas, 3-9 um diâm. Trama da lamela regular a suregular. Queilocistídios ausentes. Pleurocístidios ausentes. Basídios clavados, 14-20 × 3-4 $\mu \mathrm{m}$, tetraesporados. Basidiósporos elipsóides, hialinos, parede fina, lisa, 3,5-5,5 × 2,5-3 $\mu \mathrm{m}$.

Ilustrações: Pegler (1969, 1983a).

Material examinado: BRASIL. Mato Grosso do Sul: Rio Negro, Pantanal do Rio Negro, nas margens do Rio Correntoso, sobre madeira em decomposição, 19³0'43,6”S e 55³7’47,8”W, 24-III-2006, V.L.R. Bononi s.n. (SP381910).

A espécie tem distribuição pantropical; citada para África e vários países da América Central (Pegler 1969, 1983a). Esta é a primeira citação para o Brasil.

Tricholomopsis tropica Dennis, Trans. Br. mycol. Soc. 34: 475. 1951.

Basidioma pileado estipitado, píleo convexo, ligeiramente depresso no centro, $3 \mathrm{~cm}$ diâm. Superfície abhimenial amarela, com escamas escuras. Superfície himenial lamelar; lamelas adnatas, castanho-canela, com lamélulas; margem denteada. Estipe cilíndrico, oco, amarelo-claro, longitudinalmente estriado, com escâmulas castanhas. Trama da lamela regular. Sistema hifálico monomítico, hifas generativas hialinas, com ansas, 3-15 $\mu \mathrm{m}$ diâm. Queilocístidios piriformes, hialinos, parede fina, 30-50 × 10-25 $\mu \mathrm{m}$. Gloeocistídios abundantes, sinuosos a constritados, hialinos, conteúdo refratário, 50-60 × 3-6 $\mu \mathrm{m}$. Basídios clavados, 25$30 \mu \mathrm{m}$, tetraesporados. Basidiósporos subglobosos, hialinos, parede lisa fina, 4-5 × 3,5-5 $\mu \mathrm{m}$.

Ilustrações: Dennis (1951), Pegler (1983a).

Material examinado: BRASIL. Mato Grosso do Sul: Rio Negro, Pantanal do Rio Negro, 19³0'7,9"S e 55³6'41,9'W, sobre tronco em decomposição, 24VI-2006, V.L.R. Bononi et al. s.n. (SP381534).

Espécie de ocorrência em regiões tropicais e subtropicais (Pegler 1983a). Esta é a primeira citação da espécie para o Brasil.

Como a área do pantanal é pouco estudada os autores esperavam encontrar grande número de espécies novas, o que não ocorreu. A maioria das espécies já era conhecida de outras localidades do Brasil, ou de países vizinhos, em ambientes de cerrado, mata tropical úmida ou mesmo áreas alagadas como o chaco paraguaio. Essa observação confirma propostas de estudos florísticos que consideram o pantanal um ambiente de transição com representantes de outros ecossistemas que o circundam.

\section{Agradecimentos}

Os autores agradecem a Fundação Manoel de Barros pelo auxílio para a realização do trabalho e ao Dr. Leif Ryvarden, University of Oslo, pelas identificações de Perenniporia martii e Nigroporus macroporus.

\section{Literatura citada}

Adamoli, J. 1982. O pantanal e suas relações fitogeográficas com os cerrados. Discussão sobre o conceito de "Complexo do Pantanal". In: Anais do XXXII Congresso Nacional de Botânica. Universidade Federal do Piauí, Teresina, pp. 109-119.

Baseia,I.G. \& Milanez,A.I. 2002. Montagnea haussknechtii Rab. (Podoxales) a rare agaricoid fungus: first record from Brazil. Acta Botanica Brasilica 16: 311-315.

Bernicchia, A. 1990. Polyporaceae s.l. in Italia. Istituto di Patologia Vegetale, Università degli Studi, Bologna.

Bernicchia, A. 2000. Filobasidiella lútea a mycoparasite on Hypochnicium vellereum in Italy. Karstenia 40:49-52.

Bessette, A.E., Bessette, A.R. \& Fischer, D.W. 1996. Mushrooms of Northeastern North America. Syracuse University Press, Syracuse.

Bezerra, J.L. \& Maia, L. 2006. Filo Ascomycota. In: L.F.P. Gusmão \& L.C.Maia (eds.). Diversidade e caracterização dos fungos do Semi-Árido brasileiro. Instituto do Milênio do Semi-Árido, Recife, pp.127-139.

Bicudo, C.E.M. \& Menezes, N.A. (eds.). 1996. Biodiversity in Brazil. A first approach. CNPq, São Paulo.

Bononi, V.L.R. 1979. Basidiomicetos do Parque Estadual da Ilha do Cardoso: II. Hymenochaetaceae. Rickia 8: 85-99.

Bononi, V.L.R. 1980. Adições às espécies clavarióides, teleforóides e estereóides da Ilha do Cardoso. Rickia 9: 63-121.

Bononi, V.L.R. \& Grandi, R.A.P. (coords.). 1998. Zigomicetos, Basidiomicetos e Deuteromicetos: noções básicas de taxonomia e aplicações biotecnológicas. Instituto de Botânica, São Paulo.

Burt, E.A. 1919. The Thelephoraceae of North America XI. Tulasnella, Veluticeps, Mycobonia, Epithele and Lachnocladium. Annals of the Missouri Botanical Garden 6: 253-280.

Burt, E.A. 1920. The Thelephoraceae of North America. XII Stereum. Annals of the Missouri Botanical Garden 7: 81-240. 
Canhos, V.P. 1997. Guest Editorial: access to genetic resources and the Andean Pact. http://www.bdt. org.br/bioline/pyBiolinePublications (acesso em 15.10.2006).

Capelari, M. 1989. Agaricales do Parque Estadual da Ilha do Cardoso (exceto Tricholomataceae). Dissertação de Mestrado, Universidade de São Paulo, São Paulo.

Capelari, M. \& Gugliotta, A.M. 2005. Dactylosporina e Oudemansiella (Tricholomataceae, Oudemansiellinae) do Parque Estadual das Fontes do Ipiranga (PEFI), São Paulo, SP. Hoehnea 32: 381-387.

Capelari, M. \& Maziero, R. 1988. Fungos macroscópicos do Estado de Rondônia, Região dos Rios Jaru e JiParaná. Hoehnea 15: 28-36.

Cavalcanti, M.A.Q. 1976. Introdução ao conhecimento dos basidiomicetos poliporóides da zona da mata de Pernambuco. Tese de Livre Docência, Universidade Federal de Pernambuco, Recife.

Corner, E.J.H. 1968. A Monograph of Thelephora (Basidiomycetes). Beihefte zur Nova Hedwigia 27: 1-110.

Corner, E.J.H. 1970. Supplement to "A monograph of Clavaria and allied genera". Beihefte zur Nova Hedwigia 33: 1-299.

Corner, E.J.H. 1989. Ad Polyporaceas V. Beihefte zur Nova Hedwigia 96: 1-218.

Cunningham, G.H. 1963. The Thelephoraceae of Australia and New Zealand. Plant Deseases Division Bulletin 145: 1-359.

Da Silva, M. \& Minter, D.W. 1995. Fungi from Brazil recorded by Batista and Co-workers. Mycological Papers 169: 1-585.

Dennis, R.W.G. 1951. Some Agaricaceae of Trinidad and Venezuela. Leucosporae: Part 1. Transactions of The British Mycological Society 34: 411-482.

Dennis, R.W.G. 1970. Fungus flora of Venezuela and adjacent countries. Kew Bulletin Additional Series 3: $1-531$.

Drechsler-Santos, E.R., Groposo, C. \& Loguercio-Leite, C. 2008. Additions to the knowledge of lignocellulolytic basidiomycetes in forests from Santa Catarina, Southern Brazil. Mycotaxon 103: 197-200.

Eriksson, J. \& Ryvarden, L. 1976. The Corticiaceae of North Europe. Fungiflora, Oslo.

Eriksson, J., Hjortstam, K. \& Ryvarden, L. 1978. The Corticiaceae of North Europe. Fungiflora, Oslo.

Fidalgo, M.E.P.K. 1968a. Contribution to the fungi of Mato Grosso, Brasil. Rickia 3: 171-219.

Fidalgo, M.E.P.K. 1968b. The genus Hexagona. Memoirs of the New York Botanical Garden 17: 35-108.

Fidalgo, O. \& Bononi, V.L.R. (coords.). 1984. Técnicas de coleta, preservação e herborização de material botânico. Instituto de Botânica, São Paulo.
Fidalgo, O. \& Fidalgo, M.E.P.K. 1957. Revisão de Fungi São Paulensis. Arquivos do Museu Nacional 43: 157-188.

Fidalgo, O., Fidalgo, M.E.P.K. \& Furtado, J.S. 1965. Fungi of the "cerrado" region of São Paulo. Rickia 2: 55-71.

Fonsêca, M.P. 1999. Aphyllophorales lignocelulolíticos da Reserva Biológica do Alto da Serra de Paranapiacaba, Santo André, SP. Tese de Doutorado, Universidade de São Paulo, São Paulo.

Furtado, J.S. 1981. Taxonomy of Amauroderma (Basidiomycetes, Polyporaceae). Memoirs of the New York Botanical Garden 34: 1-109.

Gerber, A.L. \& Loguercio-Leite, C. 2000. Polyporoid wood-rotting fungi (Basiomycetes) II - New records from southern Brazil. Mycotaxon 76: 175-185.

Gibertoni, T.B. 2004. Aphyllophorales (Basidiomycotina) em áreas de Mata Atlântica do nordeste brasileiro. Tese de Doutorado, Universidade Federal de Pernambuco, Recife.

Gibertoni, T.B.\& Cavalcanti, M.A.Q. 2003. A mycological survey of the Aphyllophorales (Basidiomycotina) of the Atlantic Rain Forest in the state of Pernambuco, Brazil. Mycotaxon 87: 203-211.

Gibertoni, T.B., Parmasto, E. \& Cavalcanti, M.A.Q. 2003. Non-poroid Hymenochaetaceae (Baidiomycota) of the Atlantic Rain Forest in Northeast Brazil, with a preliminary check list of Brazilian species. Mycotaxon 87: 437-443.

Gibertoni, T.B., Ryvarden, L. \& Cavalcanti, M.A.Q. 2004. Poroid fungi (Baidiomycotina) of the Atlantic Rain Forest in the State of Pernambuco, Brazil. Synopsis Fungorum 18: 33-43.

Gibertoni, T.B., Ryvarden, L. \& Cavalcanti, M.A.Q. 2006. Stereoid fungi (Baidiomycotina) of the Atlantic Rain Forest in Northeast Brazil. Nova Hedwigia 82: 105-113.

Gilbertson, R.L. \& Budington, A.B. 1970. New records of Arizona woodrotting fungi. Journal of the ArizonaNevada Academy of Science 6: 91-97

Gilbertson, R.L. \& Ryvarden, L. 1986. North American Polypores. Fungiflora, Oslo.

Gilbertson, R.L. \& Ryvarden, L. 1987. North American Polypores. Fungiflora, Oslo.

Gimenes, L.J. 2007. A tribo Leucocoprineae (Agaricaceae) no Parque Estadual das Fontes do Ipiranga, São Paulo, SP, Brasil. Dissertação de Mestrado, Instituto de Botânica, São Paulo.

Góes Neto, A. 1999. Polypore diversity in the State of Bahia, Brazil: a historical review. Mycotaxon 72: 43-56.

Góes Neto, A. \& Baseia, I.G. 2006. Filo Basidiomycota. In: L.F.P. Gusmão \& L.C. Maia (eds.). Diversidade e caracterização dos fungos do Semi-Árido brasileiro. Instituto do Milênio do Semi-Árido, Recife, pp. 141-159. 
Góes Neto, A., Loguercio-Leite, C. \& Guerrero, R.T. 2000. Taxonomy and qualitative ecological aspects of poroid Hymenochaetales in Brazilian seasonal tropical forest. Mycotaxon 76: 197-211.

Gonçalves, G.V.C. \& Loguercio-Leite, C. 2001. Biodiversidade de fungos poróides xilófilos (Basidiomycetes), na Unidade de Conservação Ambiental Desterro (UCAD), Ilha de Santa Catarina, SC, Brasil. Insula 30: 1-19.

Gottlieb, A.M. \& Wright, J.E. 1999. Taxonomy of Ganoderma from Southern-South America: subgenus Ganoderma. Mycological Research 103: 661-673.

Gottlieb, A.M., Saidman, B.O. \& Wright, J.E. 2000. Isoenzymes of Ganoderma species from SouthernSouth America. Mycological Research 102: 415-426.

Grandi, R.A.P., Guzmán, G. \& Bononi, V.L.R. 1984. Adições às Agaricales (Basidiomycetes) do Parque Estadual das Fontes do Ipiranga, São Paulo, SP, Brasil. Rickia 11: 27-33.

Groombridge, B. 1992. Global biodiversity: status of the Earth's living resources. Chapman \& Hall, London.

Guerrero,R.T. \& Homrich,M.H. 1983. Fungos macroscópicos comuns no Rio Grande do Sul. Editora da Universidade Federal do Rio Grande do Sul, Porto Alegre.

Gugliotta, A.M. \& Bononi, V.L.R. 1999. Polyporaceae do Parque Estadual da Ilha do Cardoso, São Paulo, Brasil. Boletim do Instituto de Botânica 12: 1-112.

Guzmán, G. 1983. The Genus Psilocybe. Beihefte zur. Nova Hedwigia 74: 1-439.

Guzmán, G. 2003. Los Hongos de El Edén Quintana Roo. Introducción a la micobiota tropical de México. Conselho Nacional de Biologia, Xalapa.

Guzmán G., Bononi, V.L.R. \& Grandi, R.A.P. 1984. New species, new varieties and a new record of Psilocybe from Brazil. Mycotaxon 19: 343-350.

Guzmán-Dávalos, L. \& Guzmán, G. 1982. Contribucion al conocimento de los lepiotaceos (Fungi, Agaricales) de Quintana Roo. Boletin de la Sociedad Mexicana de Micologia 17: 43-54.

Jesus, M.A. 1993. Basidiomicetos lignocelulolíticos de floresta nativa de Pinus elliottii Engelm. do Parque Estadual das Fontes do Ipiranga, São Paulo, SP. Hoehnea 20: 119-126.

Jesus, M.A. 1996. Contribution to the knowledge of woodrotting fungi in Brazil II. Checklist of fungi from Maracá Island, Roraima State. Mycotaxon 57: 323-328.

Joly, C.A. \& Bicudo, C.E.M. (orgs.). 1998. Biodiversidade do Estado de São Paulo, Brasil: síntese do conhecimento ao final do século XX. 2: Fungos macroscópicos e plantas. FAPESP, São Paulo.

Jülich, W. 1976. Studies on hydnoid fungi I. On some genera with hyphal pegs. Persoonia 8: 447-458.
Kirk, P.M., Cannon, P.F., David, J.C. \& Stalpers, J.A. 2001. Dictionary of the Fungi. 9th ed. CAB International, Wallingford.

Loguercio-Leite, C. 1993. Polyporaceae II: Trametes Fr. na Ilha de Santa Catarina, SC, Brasil. Insula 22: 3-20.

Loguercio-Leite, C. \& Wright, J.E. 1991. Contribution to a biogeographical study of the Austro-American Polypores (Aphyllophorales) from Santa Catarina Island, SC, Brazil. Mycotaxon 40: 161-166.

Loguercio-Leite, C., Groposo, C. \& Halmenschlager, M.A. 2005. Species of Ganoderma Karsten in a subtropical area (Santa Catarina State, Southern Brazil). Iheringia, Série botânica, 60: 135-139.

Maas-Geesteranus, R.A. \& Meijer, A.A.R. 1997. Mycenae Paranaenses. Royal Netherlands Academy of Arts and Sciences, Amsterdan.

Machado, K.M.G., Matheus, D.R. \& Bononi, V.L.R. 2005. Ligninolytic enzymes production and Remazol Brilliant blue R decolorization by tropical Brazilian Basidiomycetes fungi. Brazilian Journal of Microbiology 36:246-252.

Meijer, A.A.R. 2006. Preliminary list of macromycetes from the Brazilian State of Paraná. Boletim do Museu Botânico Municipal 68: 1-55.

Mittermeier, R.A., Myers, N., Thonson, J.B., Fonseca, G.A.B. \& Olivieri, S. 1998. Hotspots and major tropical wilderness areas: approaches to setting conservation priorities. Conservation Biology 12: 516-520.

Moncalvo, J.M. \& Ryvarden, L. 1997. A nomenclatural study of the Ganodermataceae Donk. Fungiflora, Oslo.

Montoya, A., Hernandez-Totomoch, O., Estrada-Torres, A. \& Kong, A. 2003. Traditional knowledge about mushrooms in a Nahua community in the State of Tlaxcala, México. Mycologia 95: 793-806.

Nakasone, K.K. 2002. Mycoaciaella: a synonym of Phlebia. Mycotaxon 81: 477-490.

Nuñez,M.\& Ryvarden,L. 1995. Polyporus (Basidiomycotina) and related genera. Fungiflora, Oslo.

Nuñez, M. \& Ryvarden, L. 2000. East Asian Polypores. Ganodermataceae and Hymenochaetaceae. Fungiflora, Oslo.

Pegler, D.N. 1969. Studies on African Agaricales: II. Kew Bulletin 23: 219-249.

Pegler, D.N. 1983a. Agaric flora of Lesser Antilles. Kew Bulletin Additional Series 9: 1-668.

Pegler, D.N. 1983b. The genus Lentinus Fr. A world monograph. Her Majesty Service Office, London.

Pegler, D.N. 1997. The Agarics of São Paulo, Brazil. Royal Botanic Gardens, Kew.

Pereira, A.B. \& Putzke, J. 1990. Famílias e gêneros de fungos Agaricales (cogumelos) no Rio Grande do Sul. Editora das Faculdades Integradas de Santa Cruz do Sul, Santa Cruz do Sul. 
Perez-Silva, E. 1973. El genero Daldinia (Pyrenomycetes) em Mexico. Boletin de la Sociedad Mexicana de Micologia 7: 51-58.

Pott, V.J. \& Pott, A. 2000. Plantas aquáticas do Pantanal. Embrapa, Brasília.

Prance, G.T. 1973. The mycological diet of the Yanomam Indians. Mycologia 65: 248-250.

Putzke, J. \& Pereira, A.B. 1988. O gênero Oudemansiella Speg. no Rio Grande do Sul. Caderno de Pesquisa, Série botânica, 1: 47-69.

Rick, J. 1907. Contributio ad monographiam Agaricearum et Polyporacearum brasiliensium. Broteria 6: 65-92.

Rick, J. 1928. Resumo mycologico. Egatea 13: 432-439.

Rick, J. 1960. Basidiomycetes Eubasidii no Rio Grande do Sul. Brasília. 4. Meruliaceae, Polyporaceae e Boletaceae. Iheringia, Série botânica, 7: 193-295.

Rick, J. 1961. Basidiomycetes Eubasidii no Rio Grande do Sul. Brasília. 5. Agaricaceae. Iheringia, Série botânica, 8: 296-450.

Robich, G. 2006. A revised key to the species of Mycena section Fragilipedes of the northern hemisphere. Persoonia 19: 1-43.

Rogers, J.D., Ju, Y.M., Watling, R. \& Whalley, A.J.S. 1999. A reinterpretation of Daldinia concentrica based upon a recently discovered specimen. Mycotaxon 72: 507-519.

Ryvarden, L. 1984. Type studies in the Polyporaceae 16. Species described by J.M. Berkeley, either alone or with other mycologists, from 1856 to 1886 . Mycotaxon 20: 329-363.

Ryvarden, L. 2004. Neotropical Polypores: Part 1. Introduction, Ganodermataceae \& Hymenochataceae. Fungiflora, Oslo.

Ryvarden, L. \& Iturriaga, T. 2003. Studies in Neotropical polypores 10: new polypores from Venezuela. Mycologia 95: 1066-1077.

Ryvarden, L. \& Johansen, I. 1980. A preliminary flora of East Africa. Fungiflora, Oslo.

Ryvarden, L. \& Meijer, A.A.R. 2002. Studies in Neotropical polypores 14: new species from the State of Paraná, Brazil. Synopsis Fungorum 15: 34-69.

Silva, G.T. \& Gibertoni, T.B. 2006. Aphyllophorales (Basidiomycota) emáreas urbanas da Região Metropolitana do Recife, PE, Brasil. Hoehnea 33: 533-543.

Silva, M.P., Mauro, R., Mourão, G. \& Coutinho, M. 2000. Distribuição e quantificação de classes de vegetação do Pantanal através de leventamento aéreo. Revista Brasileira de Botânica 23: 143-152.

Silveira, R.M.B. \& Guerrero, R.T. 1991. Aphyllophorales poliporóides (Basidiomycetes) do Parque Nacional de Aparados da Serra, Rio Grande do Sul. Boletim do Instituto de Biociências 48:1-127.

Silveira, R.M.B. \& Wright, J.E. 2005. The taxonomy of Echinochaete and Polyporus s.str. in southern South America. Mycotaxon 93: 1-51.
Singer, R. 1961. Fungi of Northern Brazil. Publicações do Instituto de Micologia da Universidade do Recife 304: 3-26.

Singer, R. \& Digilio, A.P.L. 1952. Prodomo de la flora agaricina Argentina. Lilloa 25: 1-462.

Smith, A.H. 1947. North American species of Mycena. University of Michigan Studies-Scientific Series 17: 1-38.

Smith, A.H. 1972. The North American species of Psathyrella. Memoirs of the New York Botanical Garden 24: 1-633.

Soares, S.C.S. \& Gugliotta, A.M. 1998. Criptógamos do Parque Estadual das Fontes do Ipiranga, São Paulo, SP. Fungos, 7: Aphyllophorales (Hymenochaetaceae). Hoehnea 25: 11-31.

Sotão, H.M.P., Bononi, V.L.R. \& Figueiredo, T.S. 1991. Basidiomycetes de manguezais da Ilha de Maracá, Amapá, Brasil. Boletim do Museu Paraense Emílio Goeldi, Série botânica, 7: 109-114.

Sotão, H.M.P., Hennen, J.F., Gugliotta, A.M., Melo, O.A. \& Campos, E.L. 1997. Os Fungos Basidiomycotina. In: P.L.B. Lisboa (org.). Caxiuanã. Museu Paraense Emílio Goeldi, Belém, pp. 213-219.

Sotão, H.M.P., Campos, E.L., Costa, S.P.S.E., Melo, O.A. \& Azevedo, J.C. 2002. Basidiomycetes macroscópicos de manguezias de Bragança, Pará, Brasil. Hoehnea 29: 215-224.

Sotão, H.M.P., Campos, E.L., Gugliotta, A.M. \& Costa, S.P.S.E. 2003. Fungos Macroscópicos: Basidiomycetes. In: M.E.B. Fernandes (org.). Os manguezais da costa norte brasileira. Fundação Rio Bacanga, São Luís, pp. 45-59.

Sousa, M.A. 1980. O gênero Phellinus Quélet (Hymenochaetaceae) na Amazônia Brasileira. Tese de Doutorado, Instituto Nacional de Pesquisas da Amazônia e Fundação Universidade do Amazonas, Manaus.

Stalpers, J.A. 1996. The Aphyllophoraceous Fungii II. Keys to the Species of the Hericiales. Studies in Micology 40: 1-185. http://www.cbs.knaw.nl (acesso em 18.09.2007).

Teixeira, A.R. 1945. Himenomicetos Brasileiros. Hymeniales - Thelephoraceae. Bragantia 5: 397-434.

Teixeira, A.R. 1948. Himenomicetos brasileiros IV. Bragantia 8: 75-80.

Viégas, A.P. 1944. Alguns fungos do Brasil II. Ascomicetos. Bragantia 4: 1-392.

Vinha, P.C. 1988. Fungos macroscópicos do Estado do Espírito Santo depositados no Herbário Central da Universidade Federal do Espírito Santo, Brasil. Hoehnea 15: 57-64.

Xavier-Santos, S. 2003. Isolamento, identificação e perfil enzimático de fungos decompositores de madeira da Estação Ecológica do Noroeste Paulista - São José do Rio Preto/Mirassol, SP. Tese de Doutorado, Universidade Estadual Paulista, Rio Claro. 